\title{
Electronic Data Capture Versus Conventional Data Collection Methods in Clinical Pain Studies: Systematic Review and Meta-Analysis
}

Lindsay A Jibb ${ }^{1,2}, \mathrm{RN}, \mathrm{PhD}$; James S Khan ${ }^{3,4}$, MD, MSc; Puneet Seth ${ }^{5}$, MD; Chitra Lalloo ${ }^{1,6}, \mathrm{PhD}$; Lauren Mulrooney ${ }^{7}$, BScN; Kathryn Nicholson ${ }^{8}$, PhD; Dominik A Nowak ${ }^{5,6,9}$, MD; Harneel Kaur ${ }^{10}$, BHSc; Alyssandra Chee-A-Tow ${ }^{1}$, BSc, MPH; Joel Foster ${ }^{11}$, RN, MPA; Jennifer N Stinson ${ }^{1,2,6}$, RN-EC, PhD

${ }^{1}$ Child Health Evaluative Sciences, Hospital for Sick Children, Toronto, ON, Canada

${ }^{2}$ Lawrence S Bloomberg Faculty of Nursing, University of Toronto, Toronto, ON, Canada

${ }^{3}$ Department of Anesthesia, Mount Sinai Hospital, Toronto, ON, Canada

${ }^{4}$ Department of Anesthesia, Faculty of Medicine, University of Toronto, Toronto, ON, Canada

${ }^{5}$ Department of Family Medicine, McMaster University, Hamilton, ON, Canada

${ }^{6}$ Institute of Health Policy, Management and Evaluation, University of Toronto, Toronto, ON, Canada

${ }^{7}$ Faculty of Health Sciences, University of Ottawa, Ottawa, ON, Canada

${ }^{8}$ Department of Epidemiology and Biostatistics, Schulich School of Medicine and Dentistry, Western University, London, ON, Canada

${ }^{9}$ Department of Family and Community Medicine, Faculty of Medicine, University of Toronto, Toronto, ON, Canada

${ }^{10}$ Faculty of Medicine, University of Ottawa, Ottawa, ON, Canada

${ }^{11}$ Office of Education, Centre for Addiction and Mental Health, Toronto, ON, Canada

\section{Corresponding Author:}

Lindsay A Jibb, RN, PhD

Child Health Evaluative Sciences

Hospital for Sick Children

686 Bay Street

Toronto, ON, M5G 0A4

Canada

Phone: 14168137654 ext 309160

Email: lindsay.jibb@ @ickkids.ca

\begin{abstract}
Background: The most commonly used means to assess pain is by patient self-reported questionnaires. These questionnaires have traditionally been completed using paper-and-pencil, telephone, or in-person methods, which may limit the validity of the collected data. Electronic data capture methods represent a potential way to validly, reliably, and feasibly collect pain-related data from patients in both clinical and research settings.

Objective: The aim of this study was to conduct a systematic review and meta-analysis to compare electronic and conventional pain-related data collection methods with respect to pain score equivalence, data completeness, ease of use, efficiency, and acceptability between methods.

Methods: We searched the Medical Literature Analysis and Retrieval System Online (MEDLINE), Excerpta Medica Database (EMBASE), and Cochrane Central Register of Controlled Trials (CENTRAL) from database inception until November 2019. We included all peer-reviewed studies that compared electronic (any modality) and conventional (paper-, telephone-, or in-person-based) data capture methods for patient-reported pain data on one of the following outcomes: pain score equivalence, data completeness, ease of use, efficiency, and acceptability. We used random effects models to combine score equivalence data across studies that reported correlations or measures of agreement between electronic and conventional pain assessment methods.

Results: A total of 53 unique studies were included in this systematic review, of which 21 were included in the meta-analysis. Overall, the pain scores reported electronically were congruent with those reported using conventional modalities, with the majority of studies $(36 / 44,82 \%)$ that reported on pain scores demonstrating this relationship. The weighted summary correlation coefficient of pain score equivalence from our meta-analysis was 0.92 (95\% CI 0.88-0.95). Studies on data completeness, patientor provider-reported ease of use, and efficiency generally indicated that electronic data capture methods were equivalent or
\end{abstract}


superior to conventional methods. Most (19/23, 83\%) studies that directly surveyed patients reported that the electronic format was the preferred data collection method.

Conclusions: Electronic pain-related data capture methods are comparable with conventional methods in terms of score equivalence, data completeness, ease, efficiency, and acceptability and, if the appropriate psychometric evaluations are in place, are a feasible means to collect pain data in clinical and research settings.

(J Med Internet Res 2020;22(6):e16480) doi: 10.2196/16480

\section{KEYWORDS}

electronic; data collection; pain; efficiency; systematic review; meta-analysis

\section{Introduction}

\section{Background}

Pain is an unpleasant sensory and emotional experience that is unique to the individual. It is also a dynamic process and fluctuates in a multidimensional manner across its sensory (eg, intensity, location, duration, etc), evaluative (ie, impact on functioning) and affective (ie, emotional effect) qualities within both the short and long term [1]. Pain is influenced by a variety of biopsychosocial factors, including genetics, mood, emotions, memory, and interpersonal relationships as well as external stimuli such as physical movement [1-3]. The accurate measurement of pain is of utmost importance to clinicians and researchers.

The most commonly used methods of measuring pain within a clinical and research context are self-reported questionnaires. Clinically, pain measurements are generally performed before and after an intervention to assess a patient's response to therapy. These assessments are typically performed using paper-based questionnaires or via face-to-face or telephone-based verbal surveys or interviews. Although widely used, these conventional data collection methods can introduce a number of biases in the collected pain data. In particular, these methods often rely heavily on a patient's recall of their pain symptoms (eg, pain intensity over the preceding week). Unfortunately, the recall of pain is problematic because memories of pain are vulnerable to distortion due to physical and psychological contextual factors and selective coding and retrieval of memories [4,5]. Additional issues with conventional data collection methods include limitations in conducting ecologically valid assessments of pain in the patient's natural environment and social context, logistical challenges for repeated measurements over time, potential burden to patients, clinicians, and researchers, and possibly reduced data quality due to incomplete or back-filled pain diaries [6-8]

The advent of mobile electronic devices has created novel opportunities to collect pain-related data in clinical and research settings. Electronic data collection methods have been used to assess variables related to a variety of conditions, including mood disorders, asthma, tobacco cessation, urinary incontinence, brain injury, diabetes, cancer, and pain [7,9-11]. Specialists in pain medicine have widely advocated for the use of electronic data capture over the past two decades $[12,13]$, and mounting evidence suggests that data collected via electronic methods may be more accurate and contain fewer errors than conventional methods $[14,15]$. Although randomized controlled trials and observational studies comparing electronic and conventional data collection methods suggest benefits to the use of electronic devices in pain clinical trials, no review providing an overview of these benefits currently exists. Furthermore, with the advent of smartphone-style mobile phones and their nearly ubiquitous use in developed countries [16], electronic data collection methods are becoming more widely available. As such, a review of the literature is needed to understand the potential advantages and disadvantages of collecting pain data using electronic methods.

\section{Objective}

We aimed to identify and synthesize data from studies comparing electronic and conventional pain-related data collection methods to describe similarities and differences in pain scores, data completeness, ease of use, efficiency, and acceptability between methods.

\section{Methods}

\section{Overview}

We developed an internal protocol to guide the conduct of the review and meta-analysis. Reporting is guided by the Preferred Reporting Items for Systematic Reviews and Meta-Analyses [17].

\section{Eligibility Criteria}

\section{Criteria for Inclusion in the Systematic Review}

To be included in this review, studies must have (1) been published in English, (2) enrolled participants in a clinical study examining an acute or chronic pain-related outcome as reported by participants, (3) used both an electronic data collection method and a conventional form of data collection (ie, paper-based, telephone, or in-person), and (4) collected data on pain score equivalence (including as part of a functional limitation or disease activity measure), data completeness, ease of use, efficiency, or acceptability between collection methods. There were no restrictions on the type of study design (randomized or observational), country of study, or year of publication. Only studies in which the full texts could be retrieved were included in the review.

\section{Criteria for Inclusion in the Meta-Analysis}

A subset of studies included in the systematic review was also included in the meta-analysis. These studies reported correlations or measures of agreement (ie, intraclass correlation coefficients [ICCs], Pearson correlations, Spearman rho, and weighted kappa) between patient-reported pain intensity or pain 
interference (including affect) scores assessed using an electronic and a conventional data capture method. Pain intensity and interference were the focus of the analysis as these constructs are commonly assessed, single-item aspects of both acute and chronic pain and are routinely used to determine treatment effectiveness and guide therapy $[18,19]$. As recalled pain reports may not be an accurate reflection of the momentary pain experience, we included only studies that compared momentary pain reports. No restrictions were placed on the type of data collection method (eg, mobile phone, computer-based, and tablet), pain assessment instrument (eg, numerical rating scale [NRS]), frequency of data collection, or other pain-related assessments (ie, studies that also assessed constructs such as quality of life or disease activity in addition to pain intensity or interference were included).

\section{Study Selection}

We developed a comprehensive search strategy in consultation with a tertiary hospital librarian with expertise in the scientific literature related to digital health. We customized the search strategy to conduct tailored searches of MEDLINE, EMBASE, and Cochrane Central Register of Controlled Trials (CENTRAL) from inception until November 19, 2019. Medical Subject Headings (MeSH) keywords in the search included: pain, pain measurement, pain threshold, pain perception, electronics, cellular phone, computers, handheld, wireless technology, internet, computer communication networks, mobile applications, randomized controlled trial, multicenter study, observational study, humans, and prospective studies. Additional keywords used in the search included: pain, pain reporting, personal digital assistant, smartphone, and prospective study. An example of the search strategy can be found in Multimedia Appendix 1. We supplemented our search with searches of the author's own databases of electronic pain assessment studies.

Search results were initially electronically screened for intradatabase and interdatabase duplicates. After the electronic removal of duplicates, titles and abstracts were screened independently by 2 authors using piloted standardized screening forms (all authors involved). Subsequently, the full texts of the included citations were reviewed in duplicate to confirm study inclusion (all authors involved). The kappa statistic was calculated as a metric of screening agreement at the full-text stage. Following the literature-based precedent, we interpreted the kappa as follows: $<0.00$, poor; $0.00-0.20$, slight; 0.21-0.40, fair; 0.41-0.60, moderate; 0.61-0.80, substantial; and 0.81-1.00, almost perfect [20]. Disagreements among reviewers about study eligibility were resolved by consensus through discussion by at least three authors.

\section{Data Collection Process}

A standard data collection form was created and piloted. Data abstraction occurred independently and in duplicate. Data extracted included study design, sample size, study population, electronic and conventional data collection method, duration of data collection, score equivalence between data capture methods (ie, correlations, score differences, and descriptive reports), data completeness, ease and efficiency of data collection, and patient or participant acceptability. An a priori decision was made to not formally assess study quality given the nature of the intervention (ie, data collection method) and the diverse study designs collected in the systematic search.

\section{Data Synthesis}

Descriptive statistics (ie, frequencies and percentages) were used to synthesize and present data across all included studies. Meta-analysis was performed to synthesize results related to score equivalence across data capture methods. For the analysis, reported correlation coefficients (or kappa in the case of 2 studies [21,22]) served as effect size indices. In all studies where more than one coefficient for a correlation or measure of agreement between electronic and conventional pain data collection methods was available, we used the average of the coefficients so that a single study did not disproportionately impact the summary effect size. Whenever available, the reported sample size used to produce the score equivalence coefficient was used in the model. In cases where the sample size for the score equivalence analysis was not explicitly mentioned, we used the sample size reported for the entire study. Random-effects models were used to combine data across studies, and the $I^{2}$ statistic was used to quantify heterogeneity. The criteria set out by Higgins et al [23] were used to interpret the $I^{2}$ statistic; namely, 25\%, 50\%, and $75 \%$ were considered low, moderate, and high heterogeneity, respectively. To further examine the impact of heterogeneity on the results, the standardized residual score (ie, the standardized difference between each study effect size and the weighted mean effect size) for each study was calculated and compared [9]. A conservative cutoff of \pm 2 was set to examine extreme effect sizes as determined by the standardized residuals. We performed a sensitivity analysis to evaluate any impact of the type of correlation or measure of agreement on the weighted summary correlation. Specifically, following previously used methods, separate meta-analyses were conducted with studies reporting ICC or weighted kappa, which account for covariance and score mean and variability, and studies reporting the more conventional Pearson or Spearman rho coefficients [9]. Possible publication bias was assessed by visual inspection of an asymmetrical funnel plot. To investigate the sources of heterogeneity, we conducted further subgroup analyses. Our subgroup analyses focused on elucidating the impact of (1) the similarity of pain assessment measure between electronic and conventional modalities (ie, same measure or different) and (2) the duration of data collection (ie, once or multiple times). Subgroup analyses by study participant age and pain condition were precluded by the structure of data reported in our included studies. Meta-analysis procedures were conducted using Microsoft Excel (Microsoft Corporation) and Distiller SR Forest Plot Generator (Evidence Partners Inc).

\section{Results}

\section{Study Selection}

The search strategy identified 4927 studies, of which 183 underwent full-text review and 129 were excluded (Figure 1). The kappa agreement score between appraisers at this stage was 0.69 , which indicated substantial agreement. In all, 54 papers reporting on 53 unique studies were included in the qualitative synthesis. Stinson et al $[5,24]$ reported different results from 
the same study, so were grouped presently for analyses purposes. The number of published studies meeting our inclusion criteria In all, 21 studies were included in the quantitative synthesis. increased steadily over time (Figure 2).

Figure 1. Study selection flowchart.

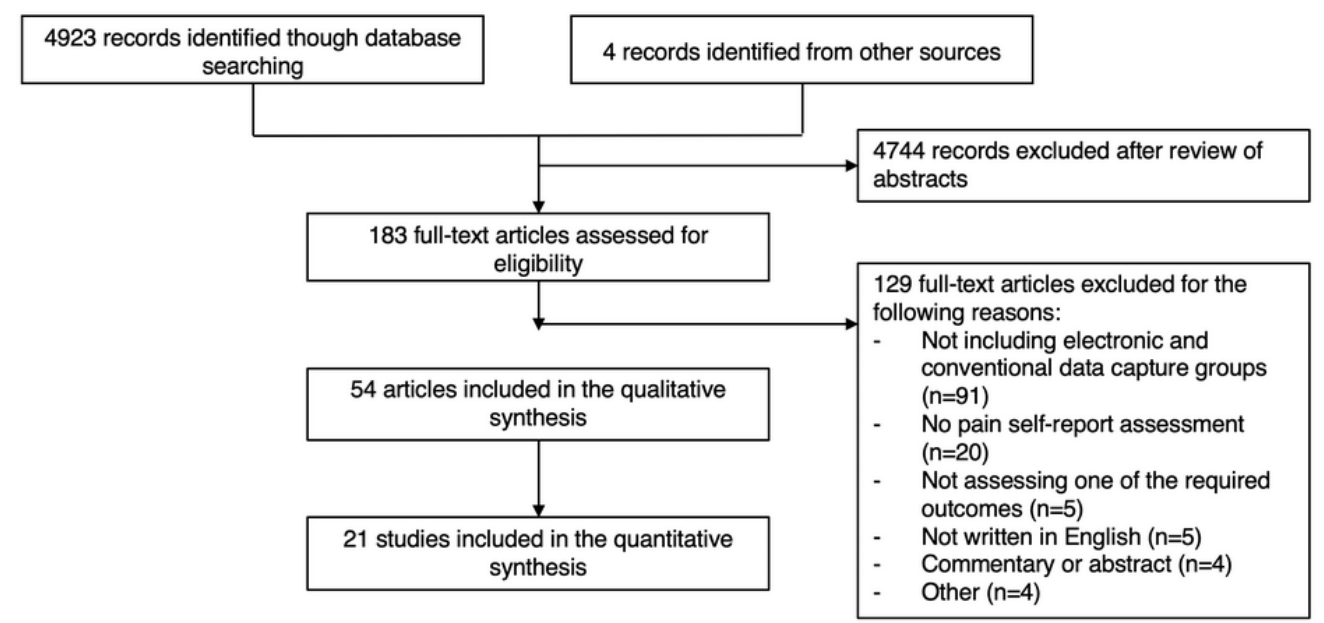

Figure 2. Number of studies meeting inclusion criteria overtime.

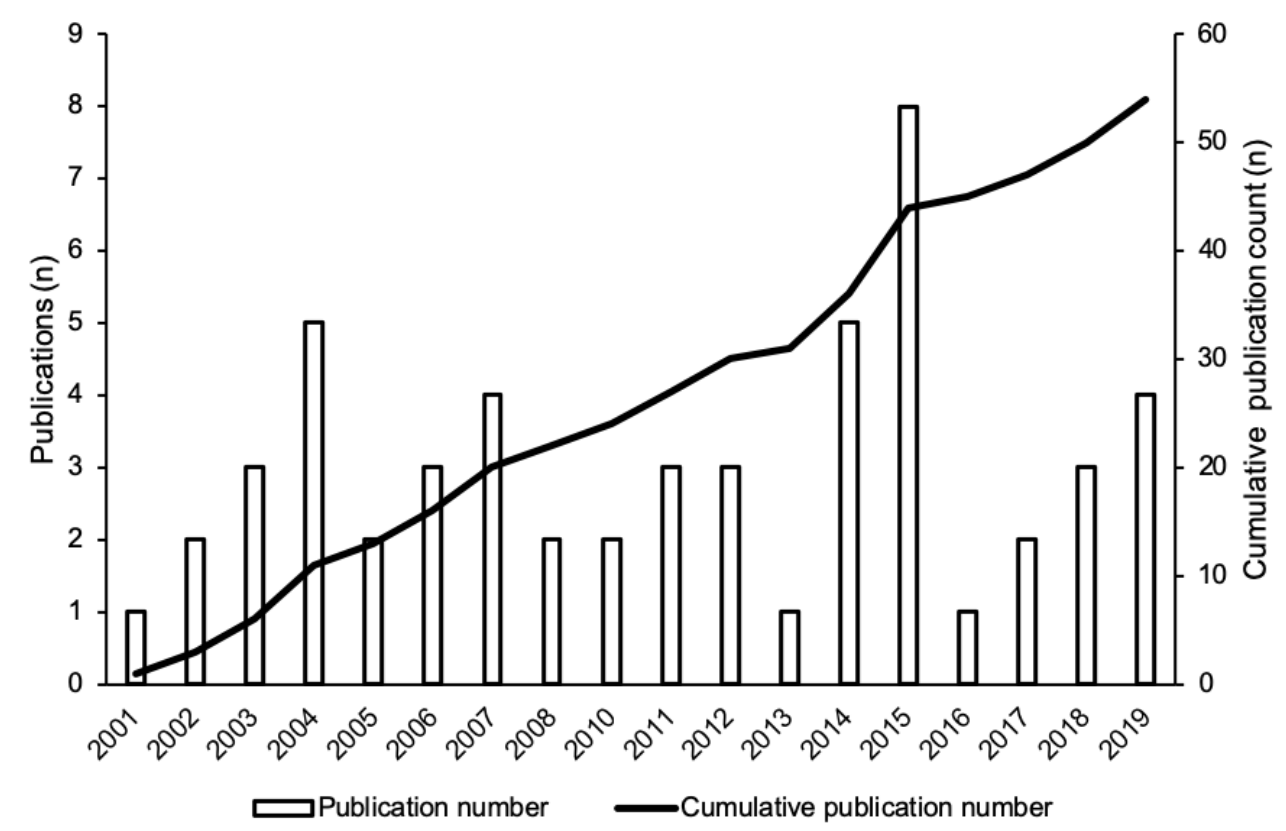

\section{Study Characteristics}

The study details are presented in Table 1. Data from a total of 7977 pain patients were included in this review. The mean number of participants across studies was 151 (range 15-2400). The average mean or median age of participants was 41.5 years (SD 17.5), and across studies, the average proportion of female participants was $63.1 \%$; mean or median age data were missing from 9 studies and sex data were missing from 7 studies. Participants in the included studies had various painful conditions or diagnoses, including both acute and chronic pain. The most common pain conditions were nonspecific chronic pain $(9 / 54,17 \%$ studies $)$, postoperative pain ( $8 / 53,15 \%$ studies $)$, and arthritis (8/53, $15 \%$ studies). 
Table 1. Study characteristics.

\begin{tabular}{|c|c|c|c|c|c|c|c|}
\hline $\begin{array}{l}\text { Authors (publica- } \\
\text { tion year) }\end{array}$ & $\begin{array}{l}\text { Criteria for } \\
\text { electronic and } \\
\text { conventional } \\
\text { pain assess- } \\
\text { ments }\end{array}$ & Study design & Sample size & $\begin{array}{l}\text { Population (age, sex, } \\
\text { pain condition) }\end{array}$ & $\begin{array}{l}\text { Electronic data } \\
\text { collection } \\
\text { modality and } \\
\text { pain data collect- } \\
\text { ed }\end{array}$ & $\begin{array}{l}\text { Conventional } \\
\text { data collection } \\
\text { method and } \\
\text { pain data collect- } \\
\text { ed }\end{array}$ & $\begin{array}{l}\text { Duration of data } \\
\text { collection }\end{array}$ \\
\hline $\begin{array}{l}\text { Allena et al (2012) } \\
\text { [25] }\end{array}$ & $\begin{array}{l}\text { Acceptability, } \\
\text { data complete- } \\
\text { ness, and ease }\end{array}$ & Not specified & 85 & $\begin{array}{l}\text { Mean age } 39.7 \text { (SD } \\
10.2 \text { ) years, } 68 \text { fe- } \\
\text { males and } 17 \text { males, } \\
\text { medication overuse } \\
\text { headache }\end{array}$ & $\begin{array}{l}\text { PDA }^{\mathrm{a}} \text { program } \\
\text { collecting data } \\
\text { on pain intensi- } \\
\text { ty (no indica- } \\
\text { tion of mea- } \\
\text { sure), pain sen- } \\
\text { sory characteris- } \\
\text { tics, associated } \\
\text { symptoms, pos- } \\
\text { sible trigger } \\
\text { factors and } \\
\text { medication use }\end{array}$ & $\begin{array}{l}\text { Paper-based } \\
\text { tool (no indica- } \\
\text { tion if questions } \\
\text { were the same } \\
\text { across formats); } \\
\text { prospective } \\
\text { recording of at- } \\
\text { tack characteris- } \\
\text { tics, more accu- } \\
\text { rate descrip- } \\
\text { tions }\end{array}$ & $\begin{array}{l}\text { Participants } \\
\text { completed both } \\
\text { formats daily } \\
\text { for 7-10 days }\end{array}$ \\
\hline $\begin{array}{l}\text { Athale et al (2004) } \\
\text { [26] }\end{array}$ & $\begin{array}{l}\text { Acceptability, } \\
\text { data complete- } \\
\text { ness, ease, and } \\
\text { score equiva- } \\
\text { lence }\end{array}$ & $\begin{array}{l}\text { Nonrandomized, } \\
\text { crossover }\end{array}$ & 43 & $\begin{array}{l}\text { Mean age not speci- } \\
\text { fied (range } 18-75+ \\
\text { years), } 36 \text { females and } \\
7 \text { males, rheumatoid } \\
\text { arthritis }\end{array}$ & $\begin{array}{l}\text { Computer pro- } \\
\text { gram collecting } \\
\text { data on } \\
\text { VAS }{ }^{\text {b }} \text {-rated } \\
\text { pain intensity, } \\
\text { pain sensory } \\
\text { characteristics, } \\
\text { and affective } \\
\text { and functional } \\
\text { impact of pain }\end{array}$ & $\begin{array}{l}\text { Paper-based } \\
\text { tool (different } \\
\text { from electronic } \\
\text { format only in } \\
\text { that pain and } \\
\text { swelling loca- } \\
\text { tions are indicat- } \\
\text { ed on separate } \\
\text { body maps) }\end{array}$ & $\begin{array}{l}\text { Participants } \\
\text { completed each } \\
\text { format once }\end{array}$ \\
\hline $\begin{array}{l}\text { Bandarian-Balooch } \\
\text { et al (2017) [27] }\end{array}$ & $\begin{array}{l}\text { Acceptability, } \\
\text { data complete- } \\
\text { ness, ease, and } \\
\text { score equiva- } \\
\text { lence }\end{array}$ & $\begin{array}{l}\text { Randomized, } \\
\text { controlled trial }\end{array}$ & 181 & $\begin{array}{l}\text { Mean age } 26.5 \text { (range } \\
18-55 \text { ) years, } 146 \mathrm{fe}- \\
\text { male and } 35 \text { males, } \\
\text { headache and mi- } \\
\text { graine }\end{array}$ & $\begin{array}{l}\text { Mobile phone } \\
\text { or computer } \\
\text { program collect- } \\
\text { ing } \mathrm{NRS}^{\mathrm{C}} \text {-rated } \\
\text { pain intensity, } \\
\text { frequency, and } \\
\text { duration data as } \\
\text { well as triggers } \\
\text { and medication } \\
\text { use }\end{array}$ & $\begin{array}{l}\text { Paper-based } \\
\text { tool with one } \\
\text { subgroup identi- } \\
\text { cal to electronic } \\
\text { format and the } \\
\text { other a long- } \\
\text { form report rep- } \\
\text { resentative of } \\
\text { conventional } \\
\text { paper diaries }\end{array}$ & $\begin{array}{l}\text { Participants } \\
\text { completed as- } \\
\text { signed format } \\
\text { once per day for } \\
30 \text { days }\end{array}$ \\
\hline $\begin{array}{l}\text { Bedson et al } \\
\text { (2019) [28] }\end{array}$ & $\begin{array}{l}\text { Data complete- } \\
\text { ness, ease, effi- } \\
\text { ciency, and } \\
\text { score equiva- } \\
\text { lence }\end{array}$ & $\begin{array}{l}\text { Nonrandomized, } \\
\text { cohort }\end{array}$ & 21 & $\begin{array}{l}\text { Median age } 62 \text { (IQR } \\
50-70) \text { years, } 13 \text { fe- } \\
\text { males and } 8 \text { males, } \\
\text { musculoskeletal pain }\end{array}$ & $\begin{array}{l}\text { Tablet program } \\
\text { collecting data } \\
\text { on NRS-rated } \\
\text { pain intensity } \\
\text { and pain inter- } \\
\text { ference, as well } \\
\text { as sleep distur- } \\
\text { bance, analgesic } \\
\text { use, mood, and } \\
\text { side effects }\end{array}$ & $\begin{array}{l}\text { Paper-based } \\
\text { tool (same as- } \\
\text { sessment as } \\
\text { used in the elec- } \\
\text { tronic study) }\end{array}$ & $\begin{array}{l}\text { Participants } \\
\text { completed elec- } \\
\text { tronic assess- } \\
\text { ment } 2 \text { times } \\
\text { per day for } 4 \\
\text { weeks and the } \\
\text { paper-based } \\
\text { tool once at } \\
\text { baseline and } \\
\text { once at study } \\
\text { completion }\end{array}$ \\
\hline $\begin{array}{l}\text { Bishop et al (2010) } \\
\text { [29] }\end{array}$ & $\begin{array}{l}\text { Acceptability, } \\
\text { data complete- } \\
\text { ness, ease, effi- } \\
\text { ciency, and } \\
\text { score equiva- } \\
\text { lence }\end{array}$ & $\begin{array}{l}\text { Randomized, } \\
\text { crossover }\end{array}$ & 167 & $\begin{array}{l}\text { Complete age data not } \\
\text { reported, (range 18- } \\
78 \text { ), complete sex data } \\
\text { not reported, back } \\
\text { pain }\end{array}$ & $\begin{array}{l}\text { Computer pro- } \\
\text { gram collecting } \\
\text { data on the oc- } \\
\text { currence of pain } \\
\text { interference } \\
\left(\mathrm{RMDQ}^{\mathrm{d}}\right)\end{array}$ & $\begin{array}{l}\text { Paper-based } \\
\text { tool (same as- } \\
\text { sessment as } \\
\text { used in the elec- } \\
\text { tronic format) }\end{array}$ & $\begin{array}{l}\text { Participants } \\
\text { completed each } \\
\text { format once in } \\
\text { random order } \\
\text { on the same day }\end{array}$ \\
\hline $\begin{array}{l}\text { Blum et al (2014) } \\
\text { [30] }\end{array}$ & $\begin{array}{l}\text { Acceptability, } \\
\text { ease, and effi- } \\
\text { ciency }\end{array}$ & $\begin{array}{l}\text { Crossover (ran- } \\
\text { domization proce- } \\
\text { dure not stated) }\end{array}$ & 62 & $\begin{array}{l}\text { Median age } 63.5 \\
\text { (range 23-86) years, } \\
31 \text { females and } 31 \\
\text { males, cancer }\end{array}$ & $\begin{array}{l}\text { PDA program } \\
\text { (E-MOSAIC) } \\
\text { collecting data } \\
\text { on VAS-rated } \\
\text { pain intensity, } \\
\text { medication use, } \\
\text { and other symp- } \\
\text { toms }\end{array}$ & $\begin{array}{l}\text { Paper-based } \\
\text { tool (same as- } \\
\text { sessment as } \\
\text { used in the elec- } \\
\text { tronic format) }\end{array}$ & $\begin{array}{l}\text { Participants } \\
\text { completed each } \\
\text { format once } \\
\text { with a 1-hour } \\
\text { washout be- } \\
\text { tween periods }\end{array}$ \\
\hline
\end{tabular}




\begin{tabular}{|c|c|c|c|c|c|c|c|}
\hline $\begin{array}{l}\text { Authors (publica- } \\
\text { tion year) }\end{array}$ & $\begin{array}{l}\text { Criteria for } \\
\text { electronic and } \\
\text { conventional } \\
\text { pain assess- } \\
\text { ments }\end{array}$ & Study design & Sample size & $\begin{array}{l}\text { Population (age, sex, } \\
\text { pain condition) }\end{array}$ & $\begin{array}{l}\text { Electronic data } \\
\text { collection } \\
\text { modality and } \\
\text { pain data collect- } \\
\text { ed }\end{array}$ & $\begin{array}{l}\text { Conventional } \\
\text { data collection } \\
\text { method and } \\
\text { pain data collect- } \\
\text { ed }\end{array}$ & $\begin{array}{l}\text { Duration of data } \\
\text { collection }\end{array}$ \\
\hline $\begin{array}{l}\text { Byrom et al (2018) } \\
\text { [31] }\end{array}$ & $\begin{array}{l}\text { Score equiva- } \\
\text { lence }\end{array}$ & $\begin{array}{l}\text { Randomized, } \\
\text { crossover }\end{array}$ & 155 & $\begin{array}{l}\text { Mean age } 48.6 \text { (SD } \\
13.1 \text { ) years (range 19- } \\
69 \text { ), } 83 \text { females and } \\
72 \text { males, chronic pain }\end{array}$ & $\begin{array}{l}\text { Mobile phone } \\
\text { or tablet pro- } \\
\text { gram collecting } \\
\text { data on VAS- } \\
\text { and NRS-relat- } \\
\text { ed pain intensi- } \\
\text { ty, as well as } \\
\text { VRS } \\
\text { pain intensity } \\
\left(\mathrm{SF}-36^{\mathrm{f}}\right)\end{array}$ & $\begin{array}{l}\text { Paper-based } \\
\text { tool (same as- } \\
\text { sessment as } \\
\text { used in the elec- } \\
\text { tronic format) }\end{array}$ & $\begin{array}{l}\text { Participants } \\
\text { completed each } \\
\text { format once } \\
\text { with a } 30 \text { - to } \\
60 \text { - min } \\
\text { washout be- } \\
\text { tween periods }\end{array}$ \\
\hline $\begin{array}{l}\text { Chiu et al (2019) } \\
\text { [32] }\end{array}$ & $\begin{array}{l}\text { Score equiva- } \\
\text { lence }\end{array}$ & $\begin{array}{l}\text { Randomized, } \\
\text { crossover }\end{array}$ & 138 & $\begin{array}{l}\text { Mean age VAS group } \\
55 \text { (SD 14) years, } 54 \\
\text { females and } 19 \text { males, } \\
\text { postoperative pain; } \\
\text { mean age NRS group } \\
53 \text { (SD 13) years, } 39 \\
\text { females and } 26 \text { males, } \\
\text { postoperative pain }\end{array}$ & $\begin{array}{l}\text { Mobile phone } \\
\text { program collect- } \\
\text { ing data on } \\
\text { VAS- and NRS- } \\
\text { rated pain inten- } \\
\text { sity }\end{array}$ & $\begin{array}{l}\text { Paper-based } \\
\text { tool (same as- } \\
\text { sessment as } \\
\text { used in the elec- } \\
\text { tronic format) }\end{array}$ & $\begin{array}{l}\text { Participants } \\
\text { completed each } \\
\text { format once } \\
\text { with a 5-min } \\
\text { washout be- } \\
\text { tween periods }\end{array}$ \\
\hline $\begin{array}{l}\text { Christie et al } \\
(2014)[33]\end{array}$ & $\begin{array}{l}\text { Data complete- } \\
\text { ness and score } \\
\text { equivalence }\end{array}$ & $\begin{array}{l}\text { Crossover (ran- } \\
\text { domization proce- } \\
\text { dure not stated) }\end{array}$ & 21 & $\begin{array}{l}\text { Median age } 49.7 \text { (SD } \\
\text { 12.2) years, } 16 \text { fe- } \\
\text { males and } 5 \text { males, in- } \\
\text { flammatory rheumatic } \\
\text { disease }\end{array}$ & $\begin{array}{l}\text { Mobile phone } \\
\text { program collect- } \\
\text { ing data on } \\
\text { NRS-rated pain } \\
\text { intensity, fa- } \\
\text { tigue, stiffness } \\
\text { and daily activi- } \\
\text { ty or function }\end{array}$ & $\begin{array}{l}\text { Paper-based } \\
\text { tool (same as- } \\
\text { sessment as } \\
\text { used in the elec- } \\
\text { tronic format) }\end{array}$ & $\begin{array}{l}\text { Participants } \\
\text { completed each } \\
\text { format on alter- } \\
\text { nate days for } 28 \\
\text { days }\end{array}$ \\
\hline $\begin{array}{l}\text { Cook et al (2004) } \\
{[34]}\end{array}$ & $\begin{array}{l}\text { Acceptability, } \\
\text { ease, and } \\
\text { score equiva- } \\
\text { lence }\end{array}$ & $\begin{array}{l}\text { Randomized, } \\
\text { crossover }\end{array}$ & 189 & $\begin{array}{l}\text { Mean age } 47.5(\mathrm{SD} \\
12.8) \text { years, } 119 \text { fe- } \\
\text { males and } 70 \text { males, } \\
\text { chronic pain }\end{array}$ & $\begin{array}{l}\text { Computer pro- } \\
\text { gram collecting } \\
\text { data on VAS- } \\
\text { and NRS-rated } \\
\text { pain intensity } \\
\text { and the affec- } \\
\text { tive impact of } \\
\text { pain (SF- } \\
\left.\text { MPQ }^{\mathrm{g}}\right) \text {. PDI } \\
\text { was also used. }\end{array}$ & $\begin{array}{l}\text { Paper-based } \\
\text { tool (same as- } \\
\text { sessment as } \\
\text { used in the elec- } \\
\text { tronic format). }\end{array}$ & $\begin{array}{l}\text { Participants } \\
\text { completed both } \\
\text { formats once } \\
\text { with a } 45 \text {-min } \\
\text { washout be- } \\
\text { tween periods }\end{array}$ \\
\hline $\begin{array}{l}\text { Cunha-Miranda et } \\
\text { al (2015) [35] }\end{array}$ & $\begin{array}{l}\text { Score equiva- } \\
\text { lence }\end{array}$ & $\begin{array}{l}\text { Nonrandomized, } \\
\text { crossover }\end{array}$ & 134 & $\begin{array}{l}\text { Mean age } 51.3(\mathrm{SD} \\
12.0) \text { years, } 100 \text { fe- } \\
\text { males and } 34 \text { males, } \\
\text { arthritis }\end{array}$ & $\begin{array}{l}\text { Tablet program } \\
\text { collecting data } \\
\text { on VAS-rated } \\
\text { pain intensity } \\
\text { and interfer- } \\
\text { ence, as well as } \\
\text { other disease } \\
\text { and quality of } \\
\text { life metrics de- } \\
\text { pendent on par- } \\
\text { ticipant diagno- } \\
\text { sis }\end{array}$ & $\begin{array}{l}\text { Paper-based } \\
\text { tool (same as- } \\
\text { sessment as } \\
\text { used in the elec- } \\
\text { tronic format). }\end{array}$ & $\begin{array}{l}\text { Participants } \\
\text { completed each } \\
\text { format with a } \\
\text { 15-min washout } \\
\text { between periods }\end{array}$ \\
\hline
\end{tabular}




\begin{tabular}{|c|c|c|c|c|c|c|c|}
\hline $\begin{array}{l}\text { Authors (publica- } \\
\text { tion year) }\end{array}$ & $\begin{array}{l}\text { Criteria for } \\
\text { electronic and } \\
\text { conventional } \\
\text { pain assess- } \\
\text { ments }\end{array}$ & Study design & Sample size & $\begin{array}{l}\text { Population (age, sex, } \\
\text { pain condition) }\end{array}$ & $\begin{array}{l}\text { Electronic data } \\
\text { collection } \\
\text { modality and } \\
\text { pain data collect- } \\
\text { ed }\end{array}$ & $\begin{array}{l}\text { Conventional } \\
\text { data collection } \\
\text { method and } \\
\text { pain data collect- } \\
\text { ed }\end{array}$ & $\begin{array}{l}\text { Duration of data } \\
\text { collection }\end{array}$ \\
\hline $\begin{array}{l}\text { Fanciullo et al } \\
(2007)[36]\end{array}$ & $\begin{array}{l}\text { Acceptability } \\
\text { and score } \\
\text { equivalence }\end{array}$ & $\begin{array}{l}\text { Crossover (ran- } \\
\text { domization proce- } \\
\text { dure not stated) }\end{array}$ & 54 & $\begin{array}{l}\text { Median age } 10.7 \text { (SD } \\
4.0 \text { ) years, } 26 \text { females } \\
\text { and } 28 \text { males, various } \\
\text { causes of pain (eg, } \\
\text { broken bones, infec- } \\
\text { tions, and cancer) }\end{array}$ & $\begin{array}{l}\text { Computer pro- } \\
\text { gram collecting } \\
\text { data on pain in- } \\
\text { tensity from an } \\
\text { investigator-de- } \\
\text { veloped comput- } \\
\text { er faces scale }\end{array}$ & $\begin{array}{l}\text { Paper-based } \\
\text { tool (Wong- } \\
\text { Baker Faces } \\
\text { Scale) }\end{array}$ & $\begin{array}{l}\text { Participants } \\
\text { completed both } \\
\text { formats once }\end{array}$ \\
\hline $\begin{array}{l}\text { Freynhagen et al } \\
(2006) \text { [37] }\end{array}$ & Ease & $\begin{array}{l}\text { Nonrandomized, } \\
\text { cohort }\end{array}$ & 717 & $\begin{array}{l}\text { Mean age } 56.0 \text { years } \\
\text { (SD not stated), sex } \\
\text { ratio not specified, } \\
\text { chronic pain }\end{array}$ & $\begin{array}{l}\text { PDA program } \\
\text { collecting data } \\
\text { on VAS-rated } \\
\text { pain intensity, } \\
\text { functional dis- } \\
\text { ability, and de- } \\
\text { pression }\end{array}$ & $\begin{array}{l}\text { Paper-based } \\
\text { tool (same as- } \\
\text { sessment as } \\
\text { used in the elec- } \\
\text { tronic format) }\end{array}$ & $\begin{array}{l}\text { Participants } \\
\text { completed ei- } \\
\text { ther format } \\
\text { once }\end{array}$ \\
\hline $\begin{array}{l}\text { Gaertner et al } \\
(2004) \text { [38] }\end{array}$ & $\begin{array}{l}\text { Acceptability, } \\
\text { data complete- } \\
\text { ness, ease, effi- } \\
\text { ciency, and } \\
\text { score equiva- } \\
\text { lence }\end{array}$ & $\begin{array}{l}\text { Randomized, } \\
\text { crossover }\end{array}$ & 24 & $\begin{array}{l}\text { Mean age } 49.9 \text { (SD } \\
15.1) \text { years, } 13 \text { fe- } \\
\text { males and } 11 \text { males, } \\
\text { various painful condi- } \\
\text { tions (eg, cancer, os- } \\
\text { teoarthritis, chronic } \\
\text { neuropathic pain) }\end{array}$ & $\begin{array}{l}\text { PDA program } \\
\text { collecting data } \\
\text { on NRS-rated } \\
\text { pain intensity, } \\
\text { analgesic use, } \\
\text { other symptoms } \\
\text { and therapies }\end{array}$ & $\begin{array}{l}\text { Paper-based } \\
\text { tool (same as- } \\
\text { sessment as } \\
\text { used in the elec- } \\
\text { tronic format) }\end{array}$ & $\begin{array}{l}\text { Participants } \\
\text { completed each } \\
\text { format daily for } \\
14 \text { days }\end{array}$ \\
\hline $\begin{array}{l}\text { Garcia-Palacios et } \\
\text { al (2013) [39] }\end{array}$ & $\begin{array}{l}\text { Acceptability, } \\
\text { data complete- } \\
\text { ness, ease, and } \\
\text { score equiva- } \\
\text { lence }\end{array}$ & $\begin{array}{l}\text { Randomized, } \\
\text { crossover }\end{array}$ & 47 & $\begin{array}{l}\text { Mean age } 48.1 \text { (SD } \\
\text { 8.0) years, } 47 \text { females, } \\
\text { fibromyalgia }\end{array}$ & $\begin{array}{l}\text { Mobile phone } \\
\text { program collect- } \\
\text { ing data on } \\
\text { NRS-rated pain } \\
\text { intensity, fa- } \\
\text { tigue, and faces } \\
\text { scale-rated } \\
\text { mood. BPI }{ }^{\mathrm{i}} \text { and } \\
\text { fatigue scale } \\
\text { were also used. }\end{array}$ & $\begin{array}{l}\text { Paper-based } \\
\text { tool (same as- } \\
\text { sessment as } \\
\text { used in the elec- } \\
\text { tronic format) }\end{array}$ & $\begin{array}{l}\text { Participants } \\
\text { completed the } \\
\text { electronic as- } \\
\text { sessment } 3 \\
\text { times per day } \\
\text { for } 1 \text { week and } \\
\text { the paper-based } \\
\text { tool once per } \\
\text { week }\end{array}$ \\
\hline $\begin{array}{l}\text { Heiberg et al } \\
(2007)[40]\end{array}$ & $\begin{array}{l}\text { Acceptability, } \\
\text { data complete- } \\
\text { ness, efficien- } \\
\text { cy, and score } \\
\text { equivalence }\end{array}$ & $\begin{array}{l}\text { Crossover (ran- } \\
\text { domization proce- } \\
\text { dure not stated) }\end{array}$ & 38 & $\begin{array}{l}\text { Mean age } 58.4 \text { (SD } \\
12.9) \text { years, } 25 \text { fe- } \\
\text { males and } 12 \text { males, } \\
\text { rheumatoid arthritis }\end{array}$ & $\begin{array}{l}\text { PDA program } \\
\text { collecting data } \\
\text { on VAS-rated } \\
\text { pain intensity, } \\
\text { fatigue, and } \\
\text { global disease } \\
\text { activity, as well } \\
\text { as NRS-rated } \\
\text { pain intensity } \\
\text { (RADAI }) \text { daily, } \\
\text { and VRS-rated } \\
\text { pain intensity } \\
\text { and interference } \\
\text { (SF-36) and ad- } \\
\text { ditional ques- } \\
\text { tions on daily } \\
\text { functioning col- } \\
\text { lected weekly }\end{array}$ & $\begin{array}{l}\text { Paper-based } \\
\text { tool (same as- } \\
\text { sessment as } \\
\text { used in the elec- } \\
\text { tronic format) }\end{array}$ & $\begin{array}{l}\text { Participants } \\
\text { completed each } \\
\text { format for } 42 \\
\text { days or } 6 \text { weeks } \\
\text { ( } 21 \text { days } 3 \\
\text { weeks for each } \\
\text { format) }\end{array}$ \\
\hline
\end{tabular}




\begin{tabular}{|c|c|c|c|c|c|c|c|}
\hline $\begin{array}{l}\text { Authors (publica- } \\
\text { tion year) }\end{array}$ & $\begin{array}{l}\text { Criteria for } \\
\text { electronic and } \\
\text { conventional } \\
\text { pain assess- } \\
\text { ments }\end{array}$ & Study design & Sample size & $\begin{array}{l}\text { Population (age, sex, } \\
\text { pain condition) }\end{array}$ & $\begin{array}{l}\text { Electronic data } \\
\text { collection } \\
\text { modality and } \\
\text { pain data collect- } \\
\text { ed }\end{array}$ & $\begin{array}{l}\text { Conventional } \\
\text { data collection } \\
\text { method and } \\
\text { pain data collect- } \\
\text { ed }\end{array}$ & $\begin{array}{l}\text { Duration of data } \\
\text { collection }\end{array}$ \\
\hline $\begin{array}{l}\text { Hofstedt et al } \\
(2019)[41]\end{array}$ & $\begin{array}{l}\text { Acceptability } \\
\text { and score } \\
\text { equivalence }\end{array}$ & $\begin{array}{l}\text { Nonrandomized, } \\
\text { cohort }\end{array}$ & 70 & $\begin{array}{l}\text { Mean age } 51.7 \text { (SD } \\
13.2 \text { ) years, } 53 \text { fe- } \\
\text { males and } 17 \text { males, } \\
\text { arthritis }\end{array}$ & $\begin{array}{l}\text { Computer, } \\
\text { tablet, or mo- } \\
\text { bile phone pro- } \\
\text { gram collecting } \\
\text { data on VAS- } \\
\text { rated pain inten- } \\
\text { sity, global } \\
\text { health, and fa- } \\
\text { tigue, as well as } \\
\text { disease activity } \\
\text { and functional } \\
\text { index for a sub- } \\
\text { set of patients }\end{array}$ & $\begin{array}{l}\text { Paper-based } \\
\text { tool (same as- } \\
\text { sessment as } \\
\text { used in the elec- } \\
\text { tronic format) }\end{array}$ & $\begin{array}{l}\text { Participants } \\
\text { completed the } \\
\text { electronic for- } \\
\text { mat at least } \\
\text { once during the } \\
\text { week before a } \\
\text { clinic appoint- } \\
\text { ment and the } \\
\text { conventional } \\
\text { format once at } \\
\text { the appointment }\end{array}$ \\
\hline $\begin{array}{l}\text { Jaatun et al (2014) } \\
\text { [42] }\end{array}$ & $\begin{array}{l}\text { Acceptability, } \\
\text { ease, score } \\
\text { equivalence }\end{array}$ & $\begin{array}{l}\text { Randomized, } \\
\text { crossover }\end{array}$ & 92 & $\begin{array}{l}\text { Age range } 20-90 \\
\text { years, } 33 \text { females and } \\
59 \text { males, cancer }\end{array}$ & $\begin{array}{l}\text { Tablet program } \\
\text { collecting data } \\
\text { on pain location } \\
\text { from an investi- } \\
\text { gator-developed } \\
\text { pain map }\end{array}$ & $\begin{array}{l}\text { Paper-based } \\
\text { tool collecting } \\
\text { pain location } \\
\text { data from the } \\
\text { BPI }\end{array}$ & $\begin{array}{l}\text { Participants } \\
\text { completed both } \\
\text { formats once a } \\
20-30 \text {-min } \\
\text { washout be- } \\
\text { tween periods }\end{array}$ \\
\hline $\begin{array}{l}\text { Jamison et al } \\
(2001)[15]\end{array}$ & $\begin{array}{l}\text { Data complete- } \\
\text { ness and score } \\
\text { equivalence }\end{array}$ & $\begin{array}{l}\text { Nonrandomized, } \\
\text { cohort }\end{array}$ & 36 & $\begin{array}{l}\text { Mean age } 42.6 \text { (SD } \\
7.0) \text { years, } 20 \text { females } \\
\text { and } 16 \text { males, chronic } \\
\text { low back pain }\end{array}$ & $\begin{array}{l}\text { PDA program } \\
\text { collecting data } \\
\text { on VAS-rated } \\
\text { pain intensity } \\
\text { each hour for } \\
16 \text { waking } \\
\text { hours as well as } \\
\text { number of sleep } \\
\text { hours }\end{array}$ & $\begin{array}{l}\text { Paper-based } \\
\text { tool collecting } \\
\text { data on NRS- } \\
\text { rated pain inten- } \\
\text { sity for each } \\
\text { waking hour } \\
\text { and telephone- } \\
\text { based NRS-pain } \\
\text { intensity over } \\
\text { the preceding } \\
\text { week }\end{array}$ & $\begin{array}{l}\text { Participants } \\
\text { completed for- } \\
\text { mats for } 1 \text { year. }\end{array}$ \\
\hline $\begin{array}{l}\text { Jamison et al } \\
(2002)[43]\end{array}$ & $\begin{array}{l}\text { Score equiva- } \\
\text { lence }\end{array}$ & $\begin{array}{l}\text { Randomized, } \\
\text { crossover }\end{array}$ & 24 & $\begin{array}{l}\text { Mean age } 34.4 \text { (range } \\
\text { 19-57) years, } 19 \text { fe- } \\
\text { males and } 5 \text { males, } \\
\text { healthy volunteers } \\
\text { holding weights heavy } \\
\text { enough to induce pain }\end{array}$ & $\begin{array}{l}\text { PDA program } \\
\text { collecting data } \\
\text { on VAS-rated } \\
\text { pain intensity }\end{array}$ & $\begin{array}{l}\text { Paper-based } \\
\text { tool (same as- } \\
\text { sessment as } \\
\text { used in the elec- } \\
\text { tronic format) }\end{array}$ & $\begin{array}{l}\text { Participants } \\
\text { completed each } \\
\text { format } 21 \text { times } \\
\text { on } 1 \text { day }\end{array}$ \\
\hline $\begin{array}{l}\text { Jamison et al } \\
(2006)[44]\end{array}$ & $\begin{array}{l}\text { Score equiva- } \\
\text { lence }\end{array}$ & $\begin{array}{l}\text { Nonrandomized, } \\
\text { cohort }\end{array}$ & 21 & $\begin{array}{l}\text { Mean age } 42.0 \text { (SD } \\
4.9) \text { years, } 9 \text { females } \\
\text { and } 12 \text { males, low } \\
\text { back pain }\end{array}$ & $\begin{array}{l}\text { PDA program } \\
\text { collecting data } \\
\text { on VAS-rated } \\
\text { pain intensity, } \\
\text { as well as the } \\
\text { affective and } \\
\text { functional im- } \\
\text { pact of pain, } \\
\text { medications, } \\
\text { and side effects }\end{array}$ & $\begin{array}{l}\text { Telephone inter- } \\
\text { views collecting } \\
\text { data on recalled } \\
\text { NRS-rated pain } \\
\text { over the previ- } \\
\text { ous week and } \\
\text { telephone-based } \\
\text { NRS-pain inten- } \\
\text { sity over the } \\
\text { preceding week }\end{array}$ & $\begin{array}{l}\text { Participants } \\
\text { completed the } \\
\text { electronic for- } \\
\text { mat at least dai- } \\
\text { ly for } 1 \text { year. }\end{array}$ \\
\hline $\begin{array}{l}\text { Jonassaint et al } \\
(2015)[45]\end{array}$ & $\begin{array}{l}\text { Score equiva- } \\
\text { lence }\end{array}$ & $\begin{array}{l}\text { Nonrandomized, } \\
\text { cohort }\end{array}$ & 15 & $\begin{array}{l}\text { Median age } 29 \text { (range } \\
\text { 16-54) years, } 6 \text { fe- } \\
\text { males and } 9 \text { males, } \\
\text { sickle cell disease }\end{array}$ & $\begin{array}{l}\text { Mobile phone } \\
\text { program collect- } \\
\text { ing VAS-rated } \\
\text { pain intensity, } \\
\text { location and } \\
\text { perceived sever- } \\
\text { ity, and treat- } \\
\text { ment strategies. }\end{array}$ & $\begin{array}{l}\text { Paper-based } \\
\text { tool collecting } \\
\text { data on VAS- } \\
\text { rated pain } \\
\text { (same assess- } \\
\text { ment as used in } \\
\text { the electronic } \\
\text { format) }\end{array}$ & $\begin{array}{l}\text { Participants } \\
\text { first completed } \\
\text { paper-based } \\
\text { tool, then elec- } \\
\text { tronic version } \\
\text { daily for } 28 \\
\text { days. }\end{array}$ \\
\hline
\end{tabular}




\begin{tabular}{|c|c|c|c|c|c|c|c|}
\hline $\begin{array}{l}\text { Authors (publica- } \\
\text { tion year) }\end{array}$ & $\begin{array}{l}\text { Criteria for } \\
\text { electronic and } \\
\text { conventional } \\
\text { pain assess- } \\
\text { ments }\end{array}$ & Study design & Sample size & $\begin{array}{l}\text { Population (age, sex, } \\
\text { pain condition) }\end{array}$ & $\begin{array}{l}\text { Electronic data } \\
\text { collection } \\
\text { modality and } \\
\text { pain data collect- } \\
\text { ed }\end{array}$ & $\begin{array}{l}\text { Conventional } \\
\text { data collection } \\
\text { method and } \\
\text { pain data collect- } \\
\text { ed }\end{array}$ & $\begin{array}{l}\text { Duration of data } \\
\text { collection }\end{array}$ \\
\hline $\begin{array}{l}\text { Junker et al (2008) } \\
\text { [46] }\end{array}$ & $\begin{array}{l}\text { Data complete- } \\
\text { ness and score } \\
\text { equivalence }\end{array}$ & $\begin{array}{l}\text { Randomized, } \\
\text { crossover }\end{array}$ & 198 & $\begin{array}{l}\text { Mean age } 56.5 \text { (SD } \\
13.9) \text { years, } 114 \text { fe- } \\
\text { males and } 84 \text { males, } \\
\text { chronic pain }\end{array}$ & $\begin{array}{l}\text { PDA program } \\
\text { collecting data } \\
\text { on VAS-rated } \\
\text { pain intensity } \\
\text { recalled pain } \\
\text { over previous } 4 \\
\text { weeks, recalled } \\
\text { worst pain in } \\
\text { previous } 4 \\
\text { weeks and a } \\
\text { summative pain } \\
\text { score }\end{array}$ & $\begin{array}{l}\text { Paper-based } \\
\text { tool (different } \\
\text { from electronic } \\
\text { format in that } \\
\text { pain intensity } \\
\text { rated on NRS) }\end{array}$ & $\begin{array}{l}\text { Participants } \\
\text { completed each } \\
\text { format once }\end{array}$ \\
\hline $\begin{array}{l}\text { Khan et al (2019) } \\
\text { [47] }\end{array}$ & $\begin{array}{l}\text { Acceptability } \\
\text { and data com- } \\
\text { pleteness }\end{array}$ & $\begin{array}{l}\text { Randomized, co- } \\
\text { hort }\end{array}$ & 78 & $\begin{array}{l}\text { Mean age } 52.7 \text { (SD } \\
11.1) \text { years, } 78 \text { fe- } \\
\text { males, postoperative } \\
\text { pain }\end{array}$ & $\begin{array}{l}\text { Computer, mo- } \\
\text { bile phone, or } \\
\text { tablet program } \\
\text { collecting data } \\
\text { on data on } \\
\text { NRS-related } \\
\text { pain intensity, } \\
\text { as well as pain } \\
\text { catastrophizing, } \\
\text { preoperative } \\
\text { anxiety, and so- } \\
\text { matic preoccu- } \\
\text { pation } \\
\text { presurgery and } \\
\text { medication use } \\
\text { and adverse } \\
\text { events post- } \\
\text { surgery }\end{array}$ & $\begin{array}{l}\text { Paper- or in- } \\
\text { person verbal } \\
\text { tool (same as- } \\
\text { sessment as } \\
\text { used in the elec- } \\
\text { tronic format) }\end{array}$ & $\begin{array}{l}\text { Participants } \\
\text { completed each } \\
\text { format twice } \\
\text { daily on postop- } \\
\text { erative days } 1 \text {, } \\
2,3 \text {, and } 9 \text { and } \\
\text { at a } 3 \text {-month } \\
\text { follow-up visit }\end{array}$ \\
\hline $\begin{array}{l}\text { Kim et al (2016) } \\
{[48]}\end{array}$ & $\begin{array}{l}\text { Acceptability } \\
\text { and efficiency }\end{array}$ & $\begin{array}{l}\text { Nonrandomized, } \\
\text { cohort }\end{array}$ & 96 & $\begin{array}{l}\text { Mean age not speci- } \\
\text { fied, } 59 \text { females and } \\
37 \text { males, spinal disor- } \\
\text { ders }\end{array}$ & $\begin{array}{l}\text { Tablet program } \\
\text { collecting data } \\
\text { on VAS-rated } \\
\text { pain intensity, } \\
\text { disability, as } \\
\text { well as ques- } \\
\text { tions related to } \\
\text { the nature of } \\
\text { pain and allevi- } \\
\text { ating and aggra- } \\
\text { vating pain fac- } \\
\text { tors }\end{array}$ & $\begin{array}{l}\text { Paper-based } \\
\text { tool (same as- } \\
\text { sessment as } \\
\text { used in electron- } \\
\text { ic format) }\end{array}$ & $\begin{array}{l}\text { Each format } \\
\text { used for a vari- } \\
\text { able and unspec- } \\
\text { ified number of } \\
\text { times }\end{array}$ \\
\hline $\begin{array}{l}\text { Koho et al (2014) } \\
\text { [49] }\end{array}$ & $\begin{array}{l}\text { Acceptability, } \\
\text { ease, and } \\
\text { score equiva- } \\
\text { lence }\end{array}$ & $\begin{array}{l}\text { Randomized, } \\
\text { crossover }\end{array}$ & 94 & $\begin{array}{l}\text { Mean age } 47.0 \text { (SD } \\
8.0) \text { years, } 55 \text { females } \\
\text { and } 39 \text { males, chronic } \\
\text { musculoskeletal pain }\end{array}$ & $\begin{array}{l}\text { Computer pro- } \\
\text { gram collecting } \\
\text { data on the af- } \\
\text { fective impact } \\
\text { of pain }\end{array}$ & $\begin{array}{l}\text { Paper-based } \\
\text { tool (same as- } \\
\text { sessment as } \\
\text { used in the elec- } \\
\text { tronic format) }\end{array}$ & $\begin{array}{l}\text { Participants } \\
\text { completed each } \\
\text { format twice on } \\
\text { two consecutive } \\
\text { days }\end{array}$ \\
\hline
\end{tabular}




\begin{tabular}{|c|c|c|c|c|c|c|c|}
\hline $\begin{array}{l}\text { Authors (publica- } \\
\text { tion year) }\end{array}$ & $\begin{array}{l}\text { Criteria for } \\
\text { electronic and } \\
\text { conventional } \\
\text { pain assess- } \\
\text { ments }\end{array}$ & Study design & Sample size & $\begin{array}{l}\text { Population (age, sex, } \\
\text { pain condition) }\end{array}$ & $\begin{array}{l}\text { Electronic data } \\
\text { collection } \\
\text { modality and } \\
\text { pain data collect- } \\
\text { ed }\end{array}$ & $\begin{array}{l}\text { Conventional } \\
\text { data collection } \\
\text { method and } \\
\text { pain data collect- } \\
\text { ed }\end{array}$ & $\begin{array}{l}\text { Duration of data } \\
\text { collection }\end{array}$ \\
\hline $\begin{array}{l}\text { Kvien et al } 2005 \\
{[50]}\end{array}$ & $\begin{array}{l}\text { Acceptability, } \\
\text { efficiency, and } \\
\text { score equiva- } \\
\text { lence }\end{array}$ & $\begin{array}{l}\text { Nonrandomized, } \\
\text { crossover }\end{array}$ & 30 & $\begin{array}{l}\text { Mean age } 61.6 \text { (range } \\
49.8-70.0 \text { ) years, } 19 \\
\text { females and } 11 \text { males, } \\
\text { rheumatoid arthritis }\end{array}$ & $\begin{array}{l}\text { PDA program } \\
\text { collecting data } \\
\text { on VAS-rated } \\
\text { pain intensity, } \\
\text { fatigue, and pa- } \\
\text { tient global } \\
\text { evaluation of } \\
\text { their disease, } \\
\text { NRS-rated pain } \\
\text { intensity } \\
\text { (RADAI), } \\
\text { VRS-rated pain } \\
\text { intensity and in- } \\
\text { terference (SF- } \\
\text { 36), and addi- } \\
\text { tional questions } \\
\text { on daily func- } \\
\text { tioning }\end{array}$ & $\begin{array}{l}\text { Paper-based } \\
\text { tool (same as- } \\
\text { sessment as } \\
\text { used in the elec- } \\
\text { tronic format) }\end{array}$ & $\begin{array}{l}\text { Participants } \\
\text { completed each } \\
\text { format on } 2 \text { oc- } \\
\text { casions } 5 \text { to } 7 \\
\text { days apart }\end{array}$ \\
\hline $\begin{array}{l}\text { MacKenzie et al } \\
(2011)[51]\end{array}$ & $\begin{array}{l}\text { Acceptability, } \\
\text { ease, efficien- } \\
\text { cy, and score } \\
\text { equivalence }\end{array}$ & $\begin{array}{l}\text { Randomized, } \\
\text { crossover }\end{array}$ & 63 & $\begin{array}{l}\text { Mean age } 53.0 \text { (range } \\
28.0-82.0 \text { ) years, } 29 \\
\text { females and } 34 \text { males, } \\
\text { psoriatic arthritis }\end{array}$ & $\begin{array}{l}\text { Computer pro- } \\
\text { gram collecting } \\
\text { data on VAS- } \\
\text { rated pain inten- } \\
\text { sity (HAQ }{ }^{\mathrm{k}} \text { ), } \\
\text { VRS-rated pain } \\
\text { intensity and in- } \\
\text { terference (SF- } \\
\text { 36) and addition- } \\
\text { al questions on } \\
\text { health and } \\
\text { arthritis-related } \\
\text { symptoms and } \\
\text { function }\end{array}$ & $\begin{array}{l}\text { Paper-based } \\
\text { tool (same as- } \\
\text { sessment as } \\
\text { used in the elec- } \\
\text { tronic format) }\end{array}$ & $\begin{array}{l}\text { Participants } \\
\text { completed each } \\
\text { format once } 1 \\
\text { hour apart }\end{array}$ \\
\hline $\begin{array}{l}\text { Marceau et al } \\
(2007)[52]\end{array}$ & $\begin{array}{l}\text { Acceptability, } \\
\text { data complete- } \\
\text { ness, ease and } \\
\text { score equiva- } \\
\text { lence }\end{array}$ & $\begin{array}{l}\text { Randomized, } \\
\text { crossover }\end{array}$ & 36 & $\begin{array}{l}\text { Mean age } 48.0 \text { (SD } \\
8.0) \text { years, } 25 \text { females } \\
\text { and } 11 \text { males, chronic } \\
\text { pain }\end{array}$ & $\begin{array}{l}\text { PDA program } \\
\text { collecting data } \\
\text { on VAS-rated } \\
\text { pain intensity } \\
\text { and interfer- } \\
\text { ence, as well as } \\
\text { on the affective } \\
\text { impact of pain, } \\
\text { medication use, } \\
\text { and pain loca- } \\
\text { tion }\end{array}$ & $\begin{array}{l}\text { Paper-based } \\
\text { tool (same as- } \\
\text { sessment as } \\
\text { used in the elec- } \\
\text { tronic format) }\end{array}$ & $\begin{array}{l}\text { Participants } \\
\text { completed each } \\
\text { format once per } \\
\text { day for } 2 \text { weeks } \\
\text { with a } 1 \text {-week } \\
\text { washout be- } \\
\text { tween periods }\end{array}$ \\
\hline $\begin{array}{l}\text { Marceau et al } \\
(2010)[53]\end{array}$ & $\begin{array}{l}\text { Acceptability } \\
\text { and ease }\end{array}$ & $\begin{array}{l}\text { Randomized, } \\
\text { controlled trial }\end{array}$ & 134 & $\begin{array}{l}\text { Mean age } 49.5 \text { (SD } \\
11.3) \text { years, } 67 \text { fe- } \\
\text { males and } 67 \text { males, } \\
\text { chronic pain }\end{array}$ & $\begin{array}{l}\text { PDA program } \\
\text { collecting data } \\
\text { on VAS-rated } \\
\text { pain intensity } \\
\text { and interfer- } \\
\text { ence, as well as } \\
\text { on the affective } \\
\text { impact of pain, } \\
\text { medication use, } \\
\text { and pain loca- } \\
\text { tion }\end{array}$ & $\begin{array}{l}\text { Paper-based } \\
\text { tool (same as- } \\
\text { sessment as } \\
\text { used in the elec- } \\
\text { tronic format) }\end{array}$ & $\begin{array}{l}\text { Participants } \\
\text { completed each } \\
\text { format monthly } \\
\text { for } 10 \text { months }\end{array}$ \\
\hline $\begin{array}{l}\text { Matthews et al } \\
\text { (2018) [54] }\end{array}$ & $\begin{array}{l}\text { Score equiva- } \\
\text { lence }\end{array}$ & $\begin{array}{l}\text { Randomized, } \\
\text { crossover }\end{array}$ & 32 & $\begin{array}{l}\text { Mean age } 24.5 \text { (SD } \\
5.6) \text { years, } 25 \text { females } \\
\text { and } 7 \text { males, nontrau- } \\
\text { matic knee pain }\end{array}$ & $\begin{array}{l}\text { Tablet-based } \\
\text { method of col- } \\
\text { lecting data on } \\
\text { pain area, loca- } \\
\text { tion, and distri- } \\
\text { bution through } \\
\text { drawing }\end{array}$ & $\begin{array}{l}\text { Paper-based } \\
\text { tool (same as- } \\
\text { sessment as } \\
\text { used in the elec- } \\
\text { tronic format) }\end{array}$ & $\begin{array}{l}\text { Participants } \\
\text { completed each } \\
\text { format once } \\
\text { with a 1-2-min } \\
\text { washout be- } \\
\text { tween periods }\end{array}$ \\
\hline
\end{tabular}




\begin{tabular}{|c|c|c|c|c|c|c|c|}
\hline $\begin{array}{l}\text { Authors (publica- } \\
\text { tion year) }\end{array}$ & $\begin{array}{l}\text { Criteria for } \\
\text { electronic and } \\
\text { conventional } \\
\text { pain assess- } \\
\text { ments }\end{array}$ & Study design & Sample size & $\begin{array}{l}\text { Population (age, sex, } \\
\text { pain condition) }\end{array}$ & $\begin{array}{l}\text { Electronic data } \\
\text { collection } \\
\text { modality and } \\
\text { pain data collect- } \\
\text { ed }\end{array}$ & $\begin{array}{l}\text { Conventional } \\
\text { data collection } \\
\text { method and } \\
\text { pain data collect- } \\
\text { ed }\end{array}$ & $\begin{array}{l}\text { Duration of data } \\
\text { collection }\end{array}$ \\
\hline $\begin{array}{l}\text { Neudecker et al } \\
(2006)[55]\end{array}$ & $\begin{array}{l}\text { Score equiva- } \\
\text { lence }\end{array}$ & $\begin{array}{l}\text { Randomized, } \\
\text { crossover }\end{array}$ & 53 & $\begin{array}{l}\text { Mean age } 51.0 \text { (range } \\
18.0-78.0 \text { ) years, } 33 \\
\text { females and } 20 \text { males, } \\
\text { postoperative pain }\end{array}$ & $\begin{array}{l}\text { PDA program } \\
\text { collecting data } \\
\text { on VAS-rated } \\
\text { pain intensity }\end{array}$ & $\begin{array}{l}\text { Manually ma- } \\
\text { nipulated slide } \\
\text { device-based } \\
\text { tool (same as- } \\
\text { sessment as } \\
\text { used in the elec- } \\
\text { tronic format) }\end{array}$ & $\begin{array}{l}\text { Participants } \\
\text { completed each } \\
\text { format while } \\
\text { participants } \\
\text { were at rest and } \\
\text { while coughing } \\
\text { (number of as- } \\
\text { sessments not } \\
\text { specified) }\end{array}$ \\
\hline $\begin{array}{l}\text { Palermo et al } \\
(2004)[56]\end{array}$ & $\begin{array}{l}\text { Acceptability, } \\
\text { data complete- } \\
\text { ness, ease, and } \\
\text { score equiva- } \\
\text { lence }\end{array}$ & $\begin{array}{l}\text { Randomized, } \\
\text { controlled trial }\end{array}$ & 60 & $\begin{array}{l}\text { Mean age electronic } \\
\text { version } 12.3 \text { (SD 2.4) } \\
\text { years, mean age paper } \\
\text { version } 12.3 \text { (SD 3.0) } \\
\text { years, } 42 \text { females and } \\
18 \text { males, headache or } \\
\text { juvenile idiopathic } \\
\text { arthritis }\end{array}$ & $\begin{array}{l}\text { PDA program } \\
\text { collecting data } \\
\text { on faces scale- } \\
\text { rated pain inten- } \\
\text { sity, pain senso- } \\
\text { ry characteris- } \\
\text { tics, affective } \\
\text { and functional } \\
\text { impact of pain }\end{array}$ & $\begin{array}{l}\text { Paper-based } \\
\text { tool (same as- } \\
\text { sessment as } \\
\text { used in the elec- } \\
\text { tronic format) }\end{array}$ & $\begin{array}{l}\text { Participants } \\
\text { completed the } \\
\text { assigned format } \\
\text { for } 7 \text { consecu- } \\
\text { tive days }\end{array}$ \\
\hline $\begin{array}{l}\text { Pawar et al (2017) } \\
\text { [57] }\end{array}$ & $\begin{array}{l}\text { Acceptability, } \\
\text { ease, efficien- } \\
\text { cy, and score } \\
\text { equivalence }\end{array}$ & $\begin{array}{l}\text { Randomized, } \\
\text { crossover }\end{array}$ & 52 & $\begin{array}{l}\text { Mean age } 46.6 \text { (SD } \\
14.5) \text { years, } 31 \text { fe- } \\
\text { males and } 21 \text { males, } \\
\text { low back pain }\end{array}$ & $\begin{array}{l}\text { Mobile phone } \\
\text { program collect- } \\
\text { ing data on the } \\
\text { occurrence of } \\
\text { pain interfer- } \\
\text { ence (RMDQ) }\end{array}$ & $\begin{array}{l}\text { Paper-based } \\
\text { tool (same as- } \\
\text { sessment as } \\
\text { used in the elec- } \\
\text { tronic format) }\end{array}$ & $\begin{array}{l}\text { Participants } \\
\text { completed each } \\
\text { format with a 1- } \\
\text { hour interval } \\
\text { between assess- } \\
\text { ments }\end{array}$ \\
\hline $\begin{array}{l}\text { Ritter et al (2004) } \\
\text { [58] }\end{array}$ & $\begin{array}{l}\text { Data complete- } \\
\text { ness and score } \\
\text { equivalence }\end{array}$ & $\begin{array}{l}\text { Randomized, } \\
\text { controlled trial }\end{array}$ & 397 & $\begin{array}{l}\text { Mean age electronic } \\
\text { version } 45.9 \text { (SD } \\
\text { 14.3) years, mean age } \\
\text { paper version } 44.6 \\
\text { (SD 13.5) years, } 287 \\
\text { females and } 110 \\
\text { males, diabetes, asth- } \\
\text { ma, heart disease, } \\
\text { lung disease, hyperten- } \\
\text { sion }\end{array}$ & $\begin{array}{l}\text { Computer pro- } \\
\text { gram collecting } \\
\text { data on } 16 \\
\text { health-related } \\
\text { variables includ- } \\
\text { ing NRS-rated } \\
\text { pain intensity }\end{array}$ & $\begin{array}{l}\text { Paper-based } \\
\text { tool (same as- } \\
\text { sessment as } \\
\text { used in the elec- } \\
\text { tronic format) }\end{array}$ & $\begin{array}{l}\text { Participants } \\
\text { completed as- } \\
\text { signed format } \\
\text { once }\end{array}$ \\
\hline $\begin{array}{l}\text { Rolfson et al } \\
(2011) \text { [59] }\end{array}$ & $\begin{array}{l}\text { Data complete- } \\
\text { ness and score } \\
\text { equivalence }\end{array}$ & $\begin{array}{l}\text { Randomized, } \\
\text { controlled trial }\end{array}$ & 2400 & $\begin{array}{l}\text { Group mean age and } \\
\text { sex ratio not specified, } \\
\text { total hip replacement } \\
\text { surgical pain }\end{array}$ & $\begin{array}{l}\text { Computer pro- } \\
\text { gram collecting } \\
\text { data on VAS- } \\
\text { rated pain inten- } \\
\text { sity and health- } \\
\text { related quality } \\
\text { of life }\end{array}$ & $\begin{array}{l}\text { Paper-based } \\
\text { tool (same as- } \\
\text { sessment as } \\
\text { used in the elec- } \\
\text { tronic format) }\end{array}$ & $\begin{array}{l}\text { Participants } \\
\text { completed as- } \\
\text { signed format } \\
\text { once }\end{array}$ \\
\hline $\begin{array}{l}\text { Saleh et al (2002) } \\
{[60]}\end{array}$ & $\begin{array}{l}\text { Acceptability } \\
\text { and score } \\
\text { equivalence }\end{array}$ & $\begin{array}{l}\text { Nonrandomized, } \\
\text { cohort }\end{array}$ & 87 & $\begin{array}{l}\text { Mean age } 63.5 \text { (SD } \\
11.6 \text { ) years, } 3 \text { females } \\
\text { and } 84 \text { males, hip or } \\
\text { knee pain }\end{array}$ & $\begin{array}{l}\text { PDA program } \\
\text { collecting data } \\
\text { on VRS-rated } \\
\text { pain intensity } \\
\text { and interference } \\
\text { (SF-36) and } \\
\text { NRS-rated pain } \\
\text { interference } \\
\text { (WOMAC })\end{array}$ & $\begin{array}{l}\text { Paper-based } \\
\text { tool (same as- } \\
\text { sessment as } \\
\text { used in the elec- } \\
\text { tronic format) }\end{array}$ & $\begin{array}{l}\text { Participants } \\
\text { completed as- } \\
\text { signed format } \\
\text { once }\end{array}$ \\
\hline $\begin{array}{l}\text { Sanchez-Rodriguez } \\
\text { et al (2015) [61] }\end{array}$ & $\begin{array}{l}\text { Acceptability } \\
\text { and score } \\
\text { equivalence }\end{array}$ & $\begin{array}{l}\text { Nonrandomized, } \\
\text { crossover }\end{array}$ & 180 & $\begin{array}{l}\text { Mean age } 14.9 \text { (SD } \\
1.64 \text {; age range: } \\
12-19) \text { years, } 104 \text { fe- } \\
\text { males and } 76 \text { males, } \\
\text { pain in the last } 3 \\
\text { months }\end{array}$ & $\begin{array}{l}\text { Mobile phone } \\
\text { program, collect- } \\
\text { ing NRS-, faces } \\
\text { pain scale-, } \\
\text { VAS-and } \\
\text { CAS }{ }^{\mathrm{m}} \text {-pain in- } \\
\text { tensity data }\end{array}$ & $\begin{array}{l}\text { Paper-based } \\
\text { tool (same as- } \\
\text { sessment as } \\
\text { used in the elec- } \\
\text { tronic format) }\end{array}$ & $\begin{array}{l}\text { Participants } \\
\text { completed each } \\
\text { assigned format } \\
\text { once with a } 30- \\
\text { min interval be- } \\
\text { tween assess- } \\
\text { ments }\end{array}$ \\
\hline
\end{tabular}




\begin{tabular}{|c|c|c|c|c|c|c|c|}
\hline $\begin{array}{l}\text { Authors (publica- } \\
\text { tion year) }\end{array}$ & $\begin{array}{l}\text { Criteria for } \\
\text { electronic and } \\
\text { conventional } \\
\text { pain assess- } \\
\text { ments }\end{array}$ & Study design & Sample size & $\begin{array}{l}\text { Population (age, sex, } \\
\text { pain condition) }\end{array}$ & $\begin{array}{l}\text { Electronic data } \\
\text { collection } \\
\text { modality and } \\
\text { pain data collect- } \\
\text { ed }\end{array}$ & $\begin{array}{l}\text { Conventional } \\
\text { data collection } \\
\text { method and } \\
\text { pain data collect- } \\
\text { ed }\end{array}$ & $\begin{array}{l}\text { Duration of data } \\
\text { collection }\end{array}$ \\
\hline $\begin{array}{l}\text { Serif et al } 2005 \\
{[62]}\end{array}$ & $\begin{array}{l}\text { Ease and effi- } \\
\text { ciency }\end{array}$ & $\begin{array}{l}\text { Nonrandomized, } \\
\text { cohort }\end{array}$ & 50 & $\begin{array}{l}\text { Age range } 27-65 \\
\text { years, sex not speci- } \\
\text { fied, back pain }\end{array}$ & $\begin{array}{l}\text { PDA program } \\
\text { collecting data } \\
\text { on VAS-pain } \\
\text { intensity, pain } \\
\text { location, and } \\
\text { other symptoms }\end{array}$ & $\begin{array}{l}\text { Paper-based } \\
\text { tool (same as- } \\
\text { sessment as } \\
\text { used in the elec- } \\
\text { tronic format) }\end{array}$ & $\begin{array}{l}\text { Participants } \\
\text { completed as- } \\
\text { sessments every } \\
2 \text { hours (be- } \\
\text { tween } 10 \text { am } \\
\text { and } 4 \text { pm) for } 5 \\
\text { days }\end{array}$ \\
\hline $\begin{array}{l}\text { Stinson et al (2008 } \\
\text { and 2014) }[5,24]\end{array}$ & $\begin{array}{l}\text { Acceptability, } \\
\text { data complete- } \\
\text { ness, ease, effi- } \\
\text { ciency, and } \\
\text { score equiva- } \\
\text { lence }\end{array}$ & $\begin{array}{l}\text { Nonrandomized, } \\
\text { cohort }\end{array}$ & $\begin{array}{l}76 \text { in non- } \\
\text { joint injec- } \\
\text { tion group } \\
\text { and } 36 \text { in } \\
\text { joint injec- } \\
\text { tion group }\end{array}$ & $\begin{array}{l}\text { Mean age nonjoint in- } \\
\text { jection group } 13.4 \\
\text { (SD 2.5) years, } 59 \text { fe- } \\
\text { males and } 17 \text { males, } \\
\text { arthritis; mean age } \\
\text { joint injection group } \\
12.6 \text { (SD 2.4) years, } \\
24 \text { females and } 12 \\
\text { males, arthritis }\end{array}$ & $\begin{array}{l}\text { PDA program } \\
\text { collecting data } \\
\text { on VAS-rated } \\
\text { pain intensity, } \\
\text { interference and } \\
\text { unpleasantness }\end{array}$ & $\begin{array}{l}\text { Paper based } \\
\text { tool (different } \\
\text { from the elec- } \\
\text { tronic tool in } \\
\text { that recall peri- } \\
\text { od was } 1 \text { week) } \\
\text { and quality of } \\
\text { life and pain } \\
\text { coping also as- } \\
\text { sessed }\end{array}$ & $\begin{array}{l}\text { Participants } \\
\text { completed the } \\
\text { electronic for- } \\
\text { mat } 3 \text { times dai- } \\
\text { ly for } 14 \text { days } \\
\text { ( } 21 \text { days for } \\
\text { joint injection } \\
\text { group) and the } \\
\text { conventional } \\
\text { format on days } \\
7 \text { and } 14 \text { (and } \\
21 \text { for joint in- } \\
\text { jection group) }\end{array}$ \\
\hline $\begin{array}{l}\text { Stinson et al } \\
\text { (2012) [63] }\end{array}$ & $\begin{array}{l}\text { Acceptability, } \\
\text { data complete- } \\
\text { ness, ease, effi- } \\
\text { ciency, and } \\
\text { score equiva- } \\
\text { lence }\end{array}$ & $\begin{array}{l}\text { Randomized, } \\
\text { crossover }\end{array}$ & $\begin{array}{l}24 \text { children } \\
\text { aged } 4-7 \\
\text { years (with } \\
\text { parents) and } \\
77 \text { youth } \\
\text { aged } 8-18 \\
\text { years }\end{array}$ & $\begin{array}{l}\text { Mean age younger } \\
\text { children } 5.9 \text { (SD 0.9) } \\
\text { years, mean age older } \\
\text { children } 13.5 \text { (SD 3.1) } \\
\text { years, } 61 \text { females and } \\
36 \text { males, various } \\
\text { rheumatic diseases }\end{array}$ & $\begin{array}{l}\text { (1) Mobile } \\
\text { phone program } \\
\text { collecting data } \\
\text { on faces scale } \\
\text { or NRS-rated } \\
\text { pain intensity, } \\
\text { pain sensory } \\
\text { characteristics } \\
\text { and affective } \\
\text { and functional } \\
\text { impact of pain } \\
\text { and (2) comput- } \\
\text { er program } \\
\text { (same assess- } \\
\text { ment as used in } \\
\text { the mobile } \\
\text { phone format) }\end{array}$ & $\begin{array}{l}\text { Paper-based } \\
\text { tool (same as- } \\
\text { sessment as } \\
\text { used in the elec- } \\
\text { tronic formats) }\end{array}$ & $\begin{array}{l}\text { Participants } \\
\text { completed each } \\
\text { format once }\end{array}$ \\
\hline $\begin{array}{l}\text { Stinson et al } \\
(2015)[7]\end{array}$ & $\begin{array}{l}\text { Acceptability, } \\
\text { data complete- } \\
\text { ness, ease, effi- } \\
\text { ciency, and } \\
\text { score equiva- } \\
\text { lence }\end{array}$ & $\begin{array}{l}\text { Nonrandomized, } \\
\text { cohort }\end{array}$ & $\begin{array}{l}92 \text { in nonsur- } \\
\text { gical group } \\
\text { and } 14 \text { in } \\
\text { surgical } \\
\text { group }\end{array}$ & $\begin{array}{l}\text { Mean age nonsurgical } \\
\text { group 13.1 (SD 2.9) } \\
\text { years, } 45 \text { females and } \\
47 \text { males, cancer; } \\
\text { mean age surgical } \\
\text { group } 14.8 \text { (SD 2.8) } \\
\text { years, } 7 \text { females and } \\
7 \text { males, cancer } \\
\text { surgery }\end{array}$ & $\begin{array}{l}\text { Mobile phone } \\
\text { program collect- } \\
\text { ing data on } \\
\text { VAS-rated pain } \\
\text { intensity, inter- } \\
\text { ference and un- } \\
\text { pleasantness, as } \\
\text { well as pain du- } \\
\text { ration and loca- } \\
\text { tion, pain man- } \\
\text { agement strate- } \\
\text { gies used }\end{array}$ & $\begin{array}{l}\text { Paper-based } \\
\text { tool (different } \\
\text { from the elec- } \\
\text { tronic tool in } \\
\text { that recall peri- } \\
\text { od was } 1 \text { week) } \\
\text { and quality of } \\
\text { life and pain } \\
\text { coping also as- } \\
\text { sessed }\end{array}$ & $\begin{array}{l}\text { Participants } \\
\text { completed the } \\
\text { electronic for- } \\
\text { mat twice daily } \\
\text { for } 14 \text { days ( } 21 \\
\text { days for surgi- } \\
\text { cal group) and } \\
\text { the convention- } \\
\text { al format on } \\
\text { days } 7 \text { and } 14 \\
\text { (and } 21 \text { for sur- } \\
\text { gical group) }\end{array}$ \\
\hline
\end{tabular}




\begin{tabular}{|c|c|c|c|c|c|c|c|}
\hline $\begin{array}{l}\text { Authors (publica- } \\
\text { tion year) }\end{array}$ & $\begin{array}{l}\text { Criteria for } \\
\text { electronic and } \\
\text { conventional } \\
\text { pain assess- } \\
\text { ments }\end{array}$ & Study design & Sample size & $\begin{array}{l}\text { Population (age, sex, } \\
\text { pain condition) }\end{array}$ & $\begin{array}{l}\text { Electronic data } \\
\text { collection } \\
\text { modality and } \\
\text { pain data collect- } \\
\text { ed }\end{array}$ & $\begin{array}{l}\text { Conventional } \\
\text { data collection } \\
\text { method and } \\
\text { pain data collect- } \\
\text { ed }\end{array}$ & $\begin{array}{l}\text { Duration of data } \\
\text { collection }\end{array}$ \\
\hline $\begin{array}{l}\text { Stomberg et al } \\
(2012)[64]\end{array}$ & $\begin{array}{l}\text { Acceptability, } \\
\text { data complete- } \\
\text { ness, ease, effi- } \\
\text { ciency, and } \\
\text { score equiva- } \\
\text { lence }\end{array}$ & $\begin{array}{l}\text { Randomized, } \\
\text { controlled trial }\end{array}$ & 40 & $\begin{array}{l}\text { Age range } 18-66 \\
\text { years, sex ratio not } \\
\text { specified, posthys- } \\
\text { terectomy and postc- } \\
\text { holecystectomy pain }\end{array}$ & $\begin{array}{l}\text { Mobile phone } \\
\text { program collect- } \\
\text { ing data on } \\
\text { NRS-rated pain } \\
\text { intensity }\end{array}$ & $\begin{array}{l}\text { Paper-based } \\
\text { tool (same as- } \\
\text { sessment as } \\
\text { used in the elec- } \\
\text { tronic format) }\end{array}$ & $\begin{array}{l}\text { Participants in } \\
\text { the electronic } \\
\text { group complet- } \\
\text { ed pain assess- } \\
\text { ments every } 4 \\
\text { hours during the } \\
\text { day for } 6 \text { days, } \\
\text { plus ad hoc re- } \\
\text { ports, partici- } \\
\text { pants in the } \\
\text { conventional } \\
\text { group complet- } \\
\text { ed pain assess- } \\
\text { ments every } 4 \\
\text { hours during the } \\
\text { day for } 4 \text { days }\end{array}$ \\
\hline $\begin{array}{l}\text { Stone et al (2003) } \\
{[65]}\end{array}$ & $\begin{array}{l}\text { Data complete- } \\
\text { ness and score } \\
\text { equivalence }\end{array}$ & $\begin{array}{l}\text { Randomized, } \\
\text { controlled trial }\end{array}$ & 91 & $\begin{array}{l}\text { Mean age across } \\
\text { groups 49.0-53.5 (SD } \\
10.4-10.7) \text { years, } 77 \\
\text { females and } 14 \text { males, } \\
\text { chronic pain }\end{array}$ & $\begin{array}{l}\text { PDA program } \\
\text { collecting data } \\
\text { on VAS-rated } \\
\text { pain intensity, } \\
\text { pain sensory } \\
\text { characteristics, } \\
\text { and affective } \\
\text { and functional } \\
\text { impact of pain }\end{array}$ & $\begin{array}{l}\text { Paper-based } \\
\text { tool (same as- } \\
\text { sessment as } \\
\text { used in the elec- } \\
\text { tronic format) }\end{array}$ & $\begin{array}{l}\text { Participants in } \\
\text { the electronic } \\
\text { group complet- } \\
\text { ed pain assess- } \\
\text { ments either } 3 \text {, } \\
6 \text {, or } 12 \text { times } \\
\text { per day for } 2 \\
\text { weeks, partici- } \\
\text { pants in the } \\
\text { conventional } \\
\text { group complet- } \\
\text { ed pain assess- } \\
\text { ments once per } \\
\text { week for } 2 \\
\text { weeks. }\end{array}$ \\
\hline $\begin{array}{l}\text { Sun et al (2015) } \\
{[66]}\end{array}$ & $\begin{array}{l}\text { Acceptability } \\
\text { and score } \\
\text { equivalence }\end{array}$ & $\begin{array}{l}\text { Randomized, } \\
\text { crossover }\end{array}$ & 128 & $\begin{array}{l}\text { Median age faces pain } \\
\text { scale group } 7.5 \text { (range } \\
\text { 4-12 years), median } \\
\text { age CAS group } 13 \\
\text { (range } 5 \text { - } 18 \text { years), } 52 \\
\text { females and } 76 \text { males, } \\
\text { postoperative pain }\end{array}$ & $\begin{array}{l}\text { Mobile phone } \\
\text { program collect- } \\
\text { ing data on } \\
\text { faces pain } \\
\text { scale- (children } \\
\text { <5 years) and } \\
\text { CAS- (children } \\
5-12 \text { years) rat- } \\
\text { ed pain intensi- } \\
\text { ty }\end{array}$ & $\begin{array}{l}\text { Paper-based } \\
\text { tool (same as- } \\
\text { sessment as } \\
\text { used in the elec- } \\
\text { tronic format) }\end{array}$ & $\begin{array}{l}\text { Participants } \\
\text { completed each } \\
\text { tool within } 10 \\
\text { min of waking } \\
\text { from surgery } \\
\text { and } 30 \text { min later } \\
\text { with a 5-min } \\
\text { washout inter- } \\
\text { val in between }\end{array}$ \\
\hline $\begin{array}{l}\text { Suso-Ribera et al } \\
(2018) \text { [67] }\end{array}$ & $\begin{array}{l}\text { Data complete- } \\
\text { ness, ease, and } \\
\text { score equiva- } \\
\text { lence }\end{array}$ & $\begin{array}{l}\text { Nonrandomized, } \\
\text { cohort }\end{array}$ & 38 & $\begin{array}{l}\text { Mean age } 42.7 \text { (SD } \\
9.9) \text { years, } 20 \text { females } \\
\text { and } 18 \text { males, chronic } \\
\text { pain }\end{array}$ & $\begin{array}{l}\text { Mobile phone- } \\
\text { based program } \\
\text { collecting data } \\
\text { on NRS-rated } \\
\text { pain intensity } \\
\text { and interfer- } \\
\text { ence, as well as } \\
\text { pain catastro- } \\
\text { phizing, pain } \\
\text { acceptance, and } \\
\text { fear and avoid- } \\
\text { ance, mood and } \\
\text { coping }\end{array}$ & $\begin{array}{l}\text { Paper- and tele- } \\
\text { phone-based } \\
\text { tool collecting } \\
\text { data on NRS- } \\
\text { rated pain inten- } \\
\text { sity and interfer- } \\
\text { ence, as well as } \\
\text { pain catastro- } \\
\text { phizing, pain } \\
\text { acceptance, and } \\
\text { fear/avoidance, } \\
\text { mood and cop- } \\
\text { ing (tools used } \\
\text { may have dif- } \\
\text { fered from elec- } \\
\text { tronic format) }\end{array}$ & $\begin{array}{l}\text { Participants } \\
\text { completed the } \\
\text { electronic for- } \\
\text { mat twice daily } \\
\text { for } 30 \text { days and } \\
\text { the convention- } \\
\text { al format at } \\
\text { baseline and af- } \\
\text { ter each study } \\
\text { week }\end{array}$ \\
\hline
\end{tabular}




\begin{tabular}{|c|c|c|c|c|c|c|c|}
\hline $\begin{array}{l}\text { Authors (publica- } \\
\text { tion year) }\end{array}$ & $\begin{array}{l}\text { Criteria for } \\
\text { electronic and } \\
\text { conventional } \\
\text { pain assess- } \\
\text { ments }\end{array}$ & Study design & Sample size & $\begin{array}{l}\text { Population (age, sex, } \\
\text { pain condition) }\end{array}$ & $\begin{array}{l}\text { Electronic data } \\
\text { collection } \\
\text { modality and } \\
\text { pain data collect- } \\
\text { ed }\end{array}$ & $\begin{array}{l}\text { Conventional } \\
\text { data collection } \\
\text { method and } \\
\text { pain data collect- } \\
\text { ed }\end{array}$ & $\begin{array}{l}\text { Duration of data } \\
\text { collection }\end{array}$ \\
\hline $\begin{array}{l}\text { Symonds et al } \\
(2015)[68]\end{array}$ & $\begin{array}{l}\text { Score equiva- } \\
\text { lence }\end{array}$ & $\begin{array}{l}\text { Nonrandomized, } \\
\text { crossover }\end{array}$ & 356 & $\begin{array}{l}\text { Mean age across } \\
\text { groups } 58.4 \text { (SD 8.4) } \\
\text { years, } 279 \text { females } \\
\text { and } 77 \text { males, os- } \\
\text { teoarthritis of the in- } \\
\text { dex knee }\end{array}$ & $\begin{array}{l}\text { PDA program } \\
\text { collecting data } \\
\text { on VRS-rated } \\
\text { pain intensity } \\
\text { and interference } \\
\text { (SF-36) and } \\
\text { NRS-rated pain } \\
\text { interference } \\
\text { (WOMAC) }\end{array}$ & $\begin{array}{l}\text { Paper-based } \\
\text { tool collected } \\
\text { data from the } \\
\text { WOMAC }\end{array}$ & $\begin{array}{l}\text { Participants } \\
\text { complete each } \\
\text { format once } \\
\text { (washout period } \\
\text { not specified) }\end{array}$ \\
\hline $\begin{array}{l}\text { Theiler et al (2007) } \\
\text { [69] }\end{array}$ & Acceptability & $\begin{array}{l}\text { Nonrandomized, } \\
\text { cohort }\end{array}$ & 60 & $\begin{array}{l}\text { Mean age } 52.1 \text { (range } \\
23.0-79.0 \text { ) years, } 36 \\
\text { females and } 24 \text { males, } \\
\text { chronic pain }\end{array}$ & $\begin{array}{l}\text { Computer pro- } \\
\text { gram collecting } \\
\text { data on NRS- } \\
\text { rated pain inten- } \\
\text { sity, medication } \\
\text { use, and other } \\
\text { symptoms }\end{array}$ & $\begin{array}{l}\text { Telephone- } \\
\text { based tool } \\
\text { (same assess- } \\
\text { ment as used in } \\
\text { the electronic } \\
\text { format) }\end{array}$ & $\begin{array}{l}\text { Participants } \\
\text { completed ei- } \\
\text { ther format ev- } \\
\text { ery day for } 1 \\
\text { week followed } \\
\text { by } 3-4 \text { days per } \\
\text { week for } 3 \text { addi- } \\
\text { tional weeks }\end{array}$ \\
\hline $\begin{array}{l}\text { VanDenKerkhof et } \\
\text { al (2003) [70] }\end{array}$ & $\begin{array}{l}\text { Data complete- } \\
\text { ness, efficien- } \\
\text { cy, and score } \\
\text { equivalence }\end{array}$ & $\begin{array}{l}\text { Nonrandomized, } \\
\text { cohort }\end{array}$ & 84 & $\begin{array}{l}\text { Age and sex ratio not } \\
\text { specified, postorthope- } \\
\text { dic surgical pain }\end{array}$ & $\begin{array}{l}\text { PDA-based pro- } \\
\text { gram collecting } \\
\text { data on NRS- } \\
\text { rated pain inten- } \\
\text { sity and physi- } \\
\text { cian orders }\end{array}$ & $\begin{array}{l}\text { Paper-based } \\
\text { tool (same as- } \\
\text { sessment as } \\
\text { used in the elec- } \\
\text { tronic format) }\end{array}$ & $\begin{array}{l}\text { Physician com- } \\
\text { pleted each for- } \\
\text { mat for half of } \\
\text { the study peri- } \\
\text { od, assessments } \\
\text { were completed } \\
\text { once per partici- } \\
\text { pant }\end{array}$ \\
\hline $\begin{array}{l}\text { VanDenKerkhof et } \\
\text { al (2004) [71] }\end{array}$ & $\begin{array}{l}\text { Data complete- } \\
\text { ness and effi- } \\
\text { ciency }\end{array}$ & $\begin{array}{l}\text { Randomized, } \\
\text { controlled trial }\end{array}$ & 74 & $\begin{array}{l}\text { Mean age electronic } \\
\text { group } 64.0 \text { (SD 10.0) } \\
\text { years, mean age con- } \\
\text { ventional group } 58.0 \\
\text { (SD 16.0) years, sex } \\
\text { ratio not specified, } \\
\text { postorthopedic surgi- } \\
\text { cal pain }\end{array}$ & $\begin{array}{l}\text { PDA program } \\
\text { collecting data } \\
\text { on NRS-rated } \\
\text { pain intensity } \\
\text { and physician } \\
\text { orders }\end{array}$ & $\begin{array}{l}\text { Paper-based } \\
\text { tool (same as- } \\
\text { sessment as } \\
\text { used in the elec- } \\
\text { tronic format) }\end{array}$ & $\begin{array}{l}\text { Participants } \\
\text { completed as- } \\
\text { signed format } \\
\text { once }\end{array}$ \\
\hline $\begin{array}{l}\text { Wæhrens et al } \\
(2015)[72]\end{array}$ & $\begin{array}{l}\text { Acceptability, } \\
\text { ease, and } \\
\text { score equiva- } \\
\text { lence }\end{array}$ & $\begin{array}{l}\text { Randomized, } \\
\text { crossover }\end{array}$ & 20 & $\begin{array}{l}\text { Mean age } 47.8 \text { (SD } \\
11.0) \text { years, } 20 \text { fe- } \\
\text { males, chronic } \\
\text { widespread pain }\end{array}$ & $\begin{array}{l}\text { Computer pro- } \\
\text { gram collecting } \\
\text { data on NRS- } \\
\text { rated pain inten- } \\
\text { sity, interfer- } \\
\text { ence, affect as } \\
\text { part of the } \\
\text { FIQ }{ }^{\mathrm{n}} \text {, as well as } \\
\text { measures of de- } \\
\text { pression, quali- } \\
\text { ty of life, cop- } \\
\text { ing and anxiety }\end{array}$ & $\begin{array}{l}\text { Paper based } \\
\text { tool (same as- } \\
\text { sessment as } \\
\text { used in the elec- } \\
\text { tronic format) }\end{array}$ & $\begin{array}{l}\text { Participants } \\
\text { completed each } \\
\text { format once } \\
\text { with a 5-min } \\
\text { wash-out inter- } \\
\text { val }\end{array}$ \\
\hline
\end{tabular}




\begin{tabular}{|c|c|c|c|c|c|c|c|}
\hline $\begin{array}{l}\text { Authors (publica- } \\
\text { tion year) }\end{array}$ & $\begin{array}{l}\text { Criteria for } \\
\text { electronic and } \\
\text { conventional } \\
\text { pain assess- } \\
\text { ments }\end{array}$ & Study design & Sample size & $\begin{array}{l}\text { Population (age, sex, } \\
\text { pain condition) }\end{array}$ & $\begin{array}{l}\text { Electronic data } \\
\text { collection } \\
\text { modality and } \\
\text { pain data collect- } \\
\text { ed }\end{array}$ & $\begin{array}{l}\text { Conventional } \\
\text { data collection } \\
\text { method and } \\
\text { pain data collect- } \\
\text { ed }\end{array}$ & $\begin{array}{l}\text { Duration of data } \\
\text { collection }\end{array}$ \\
\hline $\begin{array}{l}\text { Wood et al (2011) } \\
{[21]}\end{array}$ & $\begin{array}{l}\text { Acceptability } \\
\text { and score } \\
\text { equivalence }\end{array}$ & $\begin{array}{l}\text { Randomized, } \\
\text { crossover }\end{array}$ & 202 & $\begin{array}{l}\text { Mean age } 8.3 \text { (SD } \\
\text { 2.6) years, } 85 \text { females } \\
\text { and } 117 \text { males, postop- } \\
\text { erative or disease-relat- } \\
\text { ed pain }\end{array}$ & $\begin{array}{l}\text { PDA program } \\
\text { collecting data } \\
\text { on faces scale- } \\
\text { rated pain inten- } \\
\text { sity }\end{array}$ & $\begin{array}{l}\text { Paper-based } \\
\text { tool (same as- } \\
\text { sessment as } \\
\text { used in the elec- } \\
\text { tronic format) }\end{array}$ & $\begin{array}{l}\text { Participants } \\
\text { completed each } \\
\text { format once } \\
\text { with a } 30-\text { min } \\
\text { washout be- } \\
\text { tween periods }\end{array}$ \\
\hline
\end{tabular}

${ }^{\text {a} P D A: ~ p e r s o n a l ~ d i g i t a l ~ a s s i s t a n t . ~}$

${ }^{b}$ VAS: Visual Analog Scale.

${ }^{\mathrm{c}}$ NRS: Numerical Rating Scale.

${ }^{\mathrm{d}}$ RMDQ: Roland Morris Disability Questionnaire.

eVRS: Verbal Rating Scale.

${ }^{f}$ SF-36: Short Form 36 Health Survey.

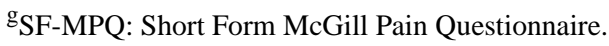

${ }^{\mathrm{h}}$ PDI: Pain Disability Index.

${ }^{\mathrm{i}}$ BPI: Brief Pain Inventory.

${ }^{\mathrm{j}}$ RADAI: Rheumatoid Arthritis Disease Activity Index.

${ }^{\mathrm{k}} \mathrm{HAQ}$ : Health Assessment Questionnaire.

${ }^{1}$ WOMAC: Western Ontario and McMaster University Osteoarthritis Index.

${ }^{\mathrm{m}} \mathrm{CAS}$ : Color Analogue Scale.

${ }^{\mathrm{n}}$ FIQ: Fibromyalgia Impact Questionnaire.

Regarding electronic data capture modalities, the devices used for data collection included the following: personal digital assistants (PDA; 22/53, 41\%), computer (either Web-based or offline; 10/53, 18\%), smartphone $(9 / 53,17 \%)$, tablet $(5 / 53,9 \%)$, mobile phones, tablets, and//or computers $(6 / 53,11 \%)$, and conventional mobile phone $(1 / 53,22 \%)$. Studies conducted more recently tended to use non-PDA-based mobile modalities, whereas older studies utilized PDA and computer-based modalities of assessment (average year of publication for studies employing non-PDA mobile devices was 2016 versus 2007 for studies on PDA and computer-based modalities). Conventional pain assessment modalities were paper-based $(46 / 53,86.7 \%)$, telephone-interviews $(2 / 53 ; 43 \%)$, paper- and verbal-based $(3 / 53,65 \%)$, face-to-face interviews $(1 / 53,22 \%)$, and a manually manipulated slide device $(1 / 53,22 \%)$.

In total, 35\% (19/53) studies used a randomized, crossover design, $14(26 \%)$ studies used a nonrandomized cohort design, $9(17 \%)$ studies were randomized controlled trials, $5(9 \%)$ studies used a nonrandomized crossover design, 5 (9\%) studies used a crossover design with unclear randomization (no mention of whether a randomization procedure was employed), and 1 (22\%) study did not specify the study design. The duration of data collection varied across studies, ranging from a single assessment being conducted to repeated assessments over the course of a year.

\section{Data Related to Pain Assessment Measures}

Pain intensity was the most commonly assessed pain outcome, measured in $90 \%$ (48/53) of studies. Methods to measure pain intensity using electronic methods were visual analog scales
(VAS; 26/53, 49\%), NRS (22/53, 41\%), faces scales $(5 / 53,9 \%)$, verbal rating scales $(5 / 53,9 \%)$, and color analogue scales $(2 / 53$, $44 \%$ ). The method of pain intensity measurement was not specified in 1 study (21.9\%). In total, 75\% (40/53) of studies employed the same measurement tools across the electronic and conventional modalities.

Pain assessment tools using electronic data capture most often were multidimensional in nature $(35 / 53,66 \%)$. Electronic data collection methods were used to capture multidimensional aspects of pain using the following validated questionnaires: Brief Pain Inventory, Fibromyalgia Impact Questionnaire, Health Assessment Questionnaire, Pain Disability Index, Rheumatoid Arthritis Disease Activity Index, Roland-Morris Disability Questionnaire, Short Form 20, Short Form 36, Short Form McGill Pain Questionnaire, and Western Ontario and McMaster Universities Arthritis Index.

\section{Comparisons Across Data Collection Modalities}

\section{Qualitative Synthesis of Score Equivalence}

In total, $83 \%(44 / 53)$ of studies reported pain score equivalence between electronic and conventional data capture methods (Table 2). Statistical methods used to compare scores differed between studies: $47 \%$ (21/44) of these studies used correlational analyses (ie, ICC, Pearson coefficient, Spearman coefficient, or weighted kappa) to examine the agreement between pain scores; $29 \%$ (13/44) studies statistically examined the differences between mean or median score, SDs, or ranges between methods; $76 \%$ (3/44) studies used descriptive methods to examine agreement; and $15 \%$ (7/44) studies used a combination of these statistical methods. 
Table 2. Summary of study results related to score equivalence.

\begin{tabular}{|c|c|c|c|c|c|}
\hline \multirow{3}{*}{$\begin{array}{l}\text { Outcome and study } \\
\text { (year) }\end{array}$} & \multicolumn{5}{|c|}{ Equivalence examination method and results } \\
\hline & \multicolumn{2}{|l|}{ Score correlation } & \multicolumn{2}{|l|}{ Score differences } & \multirow[t]{2}{*}{ Descriptive } \\
\hline & Method & Results & Method & Results & \\
\hline \multicolumn{6}{|c|}{ Studies reporting pain score equivalence } \\
\hline $\begin{array}{l}\text { Athale et al } \\
\text { (2004) [26] }\end{array}$ & $\mathrm{ICC}^{\mathrm{a}}$ & $\begin{array}{l}\text { Pain intensity } \\
\text { ICC }=0.941 ; \text { pain inter- } \\
\text { ference } \mathrm{ICC}=0.959\end{array}$ & $-^{b}$ & - & - \\
\hline $\begin{array}{l}\text { Bandarian- } \\
\text { Balooch et al } \\
(2017)[27]\end{array}$ & - & - & ANOVA $^{\mathrm{c}}$ & $\begin{array}{l}\text { Mean pain intensity, } \\
\text { frequency, duration, } \\
\text { medication usage, dis- } \\
\text { ability } P>.05 \text { of all }\end{array}$ & - \\
\hline $\begin{array}{l}\text { Bishop et al } \\
\text { (2010) [29] }\end{array}$ & ICC & $\begin{array}{l}\text { Pain interference } \\
\text { ICC }=0.965\end{array}$ & - & - & $\begin{array}{l}\text { Mean low-back pain interference } \\
\text { score difference between method } \\
0.03 \text { (SD } 1.43 ; 95 \% \text { CI }-0.19 \text { to } \\
0.25 \text { ). Authors predefined accept- } \\
\text { able } 95 \% \text { CI was } \pm 0.5 \text {. }\end{array}$ \\
\hline $\begin{array}{l}\text { Byrom et al } \\
\text { (2018) [31] }\end{array}$ & ICC & $\begin{array}{l}\text { Pain intensity } r=0.87 \text { - } \\
0.98,95 \% \text { CI } 0.83- \\
0.99)\end{array}$ & - & - & - \\
\hline $\begin{array}{l}\text { Castarlanas et al } \\
\text { (2015) [22] }\end{array}$ & Weighted kappa & Pain intensity $\kappa=0.813$ & - & - & - \\
\hline $\begin{array}{l}\text { Chiu et al (2019) } \\
\text { [32] }\end{array}$ & $\begin{array}{l}\text { Pearson correla- } \\
\text { tion }\end{array}$ & $\begin{array}{l}\text { Pain intensity } r=0.93 \text { - } \\
0.96(P<.001)\end{array}$ & - & - & $\begin{array}{l}\text { Using Bland-Altman method, an } \\
\text { agreement between the data capture } \\
\text { techniques shown at } 95 \% \text { CI. }\end{array}$ \\
\hline $\begin{array}{l}\text { Christie et al } \\
\text { (2014) [33] }\end{array}$ & - & - & $\begin{array}{l}\text { Paired sample } t \\
\text { tests or Wilcoxon } \\
\text { Signed Rank Test }\end{array}$ & $\begin{array}{l}\text { Mean, SD, and range } \\
\text { of pain intensity } \\
P>.46 \text { for all }\end{array}$ & - \\
\hline $\begin{array}{l}\text { Cook et al (2004) } \\
{[34]}\end{array}$ & Spearman rho & $\begin{array}{l}\text { Pain intensity and in- } \\
\text { terference rho=0.67- } \\
084\end{array}$ & - & - & - \\
\hline $\begin{array}{l}\text { Cunha Miranda } \\
\text { et al (2015) [35] }\end{array}$ & ICC & $\begin{array}{l}\text { Pain intensity and in- } \\
\text { terference } \\
\text { ICC }=>0.781-0.944\end{array}$ & - & - & - \\
\hline $\begin{array}{l}\text { Fanciullo et al } \\
\text { (2007) [36] }\end{array}$ & Spearman rho & $\begin{array}{l}\text { Pain intensity } \\
\text { rho }=-0.72(P<.001)\end{array}$ & - & - & - \\
\hline $\begin{array}{l}\text { Gaertner et al } \\
\text { (2004) [38] }\end{array}$ & - & - & $t$ test & $\begin{array}{l}\text { Mean pain intensity } \\
\text { not significantly differ- } \\
\text { ent }(P \text { value not report- } \\
\text { ed) }\end{array}$ & - \\
\hline $\begin{array}{l}\text { Garcia-Palacios } \\
\text { et al (2013) [39] }\end{array}$ & $\begin{array}{l}\text { Pearson correla- } \\
\text { tion }\end{array}$ & $\begin{array}{l}\text { Pain intensity } r=0.79 \\
(P<.001)\end{array}$ & - & - & - \\
\hline $\begin{array}{l}\text { Heiberg et al } \\
(2007) \text { [40] }\end{array}$ & - & - & $\begin{array}{l}\text { Wilcoxon's signed } \\
\text { rank test }\end{array}$ & $\begin{array}{l}\text { Mean, SD, and range } \\
\text { of pain intensity } \\
P>.06\end{array}$ & - \\
\hline $\begin{array}{l}\text { Hofstedt et al } \\
\text { (2019) [41] }\end{array}$ & ICC & $\begin{array}{l}\text { Pain intensity } \\
\text { ICC }=0.952\end{array}$ & Paired $t$ test & $\begin{array}{l}\text { Mean pain intensity } \\
\text { not significantly differ- } \\
\text { ent }(P=.29)\end{array}$ & $\begin{array}{l}\text { Using Bland-Altman method, an } \\
\text { agreement between the data capture } \\
\text { techniques shown at } 95 \% \text { CI. }\end{array}$ \\
\hline $\begin{array}{l}\text { Jaatun et al } \\
\text { (2014) [42] }\end{array}$ & - & - & - & - & $\begin{array}{l}\text { In } 71 \%(65 / 92) \text { of cases participants } \\
\text { marked the same number of areas } \\
\text { and the same anatomical locations } \\
\text { on both body map versions, in } 20 \\
\text { cases, the markings were relatively } \\
\text { similar, and in } 7 \text { cases, the markings } \\
\text { were dissimilar. }\end{array}$ \\
\hline
\end{tabular}




\begin{tabular}{|c|c|c|c|c|c|}
\hline \multirow{3}{*}{$\begin{array}{l}\text { Outcome and study } \\
\text { (year) }\end{array}$} & \multicolumn{5}{|c|}{ Equivalence examination method and results } \\
\hline & \multicolumn{2}{|l|}{ Score correlation } & \multicolumn{2}{|l|}{ Score differences } & \multirow[t]{2}{*}{ Descriptive } \\
\hline & Method & Results & Method & Results & \\
\hline $\begin{array}{l}\text { Jamison et al } \\
\text { (2001) [15] }\end{array}$ & $\begin{array}{l}\text { Pearson correla- } \\
\text { tion }\end{array}$ & $\begin{array}{l}\text { Pain intensity } r=0.88 \text {, } \\
P<.001\end{array}$ & - & - & - \\
\hline $\begin{array}{l}\text { Jamison et al } \\
\text { (2002) [43] }\end{array}$ & $\begin{array}{l}\text { Pearson correla- } \\
\text { tion }\end{array}$ & $\begin{array}{l}\text { Pain intensity } \\
r^{2}>0.999\end{array}$ & - & - & - \\
\hline $\begin{array}{l}\text { Jamison et al } \\
\text { (2006) [44] }\end{array}$ & $\begin{array}{l}\text { Pearson correla- } \\
\text { tion }\end{array}$ & $\begin{array}{l}\text { Pain intensity } r=0.99 \\
(95 \% \text { CI } 0.975-0.996)\end{array}$ & - & - & - \\
\hline $\begin{array}{l}\text { Jonassaint et al } \\
\text { (2015) [45] }\end{array}$ & ICC & $\begin{array}{l}\text { Pain intensity } \\
\text { ICC }=0.97(95 \% \text { CI } \\
0.88-0.99)\end{array}$ & - & - & - \\
\hline $\begin{array}{l}\text { Kvien et al } \\
(2005)[50]\end{array}$ & $\begin{array}{l}\text { Pearson correla- } \\
\text { tion }\end{array}$ & $\begin{array}{l}\text { Pain intensity } r=0.79 \text { - } \\
0.93\end{array}$ & - & - & - \\
\hline $\begin{array}{l}\text { MacKenzie et al } \\
\text { (2011) [51] }\end{array}$ & ICC & $\begin{array}{l}\text { Pain intensity and in- } \\
\text { terference ICC }=0.95- \\
0.97 ; 95 \% \text { CI } 0.95- \\
0.98)\end{array}$ & - & - & - \\
\hline $\begin{array}{l}\text { Marceau et al } \\
\text { (2007) [52] }\end{array}$ & - & - & - & - & $\begin{array}{l}\text { Participants reported similar using } \\
\text { each data capture methods for pain } \\
\text { intensity, pain interference, mood, } \\
\text { and helpfulness of medications. }\end{array}$ \\
\hline $\begin{array}{l}\text { Matthews et al } \\
\text { (2018) [54] }\end{array}$ & $\begin{array}{l}\text { Pearson correla- } \\
\text { tion and ICC }\end{array}$ & $\begin{array}{l}\text { Pain location pixelat- } \\
\text { ed area } r=0.93 \\
(P<.001) \text { and } \\
\text { ICC }=0.966(P<.001)\end{array}$ & $t$ test & $\begin{array}{l}\text { Mean pain location } \\
\text { pixelated area not sig- } \\
\text { nificantly different } \\
(P=.93)\end{array}$ & $\begin{array}{l}\text { Using Bland-Altman method, an } \\
\text { agreement between the data capture } \\
\text { techniques shown at } 95 \% \text { CI. }\end{array}$ \\
\hline $\begin{array}{l}\text { Neudecker et al } \\
\text { (2006) [55] }\end{array}$ & $\begin{array}{l}\text { Pearson correla- } \\
\text { tion }\end{array}$ & $\begin{array}{l}\text { Pain intensity } r=0.902 \\
(P<.001)\end{array}$ & - & - & - \\
\hline $\begin{array}{l}\text { Palermo et al } \\
\text { (2004) [56] }\end{array}$ & - & - & $t$ test & $\begin{array}{l}\text { Mean pain intensity } \\
\text { not significantly differ- } \\
\text { ent }(P \text { value not report- } \\
\text { ed) }\end{array}$ & - \\
\hline $\begin{array}{l}\text { Pawar et al } \\
\text { (2017) [57] }\end{array}$ & ICC & $\begin{array}{l}\text { Pain interference } \\
\text { ICC }=0.994(95 \% \text { CI } \\
0.989-0.996)\end{array}$ & - & - & - \\
\hline $\begin{array}{l}\text { Ritter et al (2004) } \\
\text { [58] }\end{array}$ & - & - & $\begin{array}{l}t \text { test, Wilcoxon's } \\
\text { signed rank test } \\
\text { and ANCOVA }\end{array}$ & $\begin{array}{l}\text { Mean pain intensity } \\
\text { and pain interference } \\
P>.30\end{array}$ & - \\
\hline $\begin{array}{l}\text { Saleh et al (2002) } \\
{[60]}\end{array}$ & - & - & Test not reported & $\begin{array}{l}\text { Mean and SD pain in- } \\
\text { tensity and interfer- } \\
\text { ence not significantly } \\
\text { different ( } P \text { value not } \\
\text { reported) }\end{array}$ & - \\
\hline $\begin{array}{l}\text { Sanchez-Ro- } \\
\text { driguez et al } \\
\text { (2015) [61] }\end{array}$ & - & - & - & - & $\begin{array}{l}\text { Using Bland-Altman method, an } \\
\text { agreement between the data capture } \\
\text { techniques shown for the FPS-R }{ }^{\mathrm{e}} \text {, } \\
\text { the VAS }{ }^{\mathrm{f}} \text {, and the CAS }{ }^{\mathrm{g}} \text { at } 95 \% \text { CI. } \\
\text { Agreement for the } \mathrm{NRS}^{\mathrm{h}}-11 \text { shown } \\
\text { in the } 80 \% \text { CI level. }\end{array}$ \\
\hline $\begin{array}{l}\text { Stinson et al } \\
\text { (2012) [63] }\end{array}$ & - & - & $t$ test & $\begin{array}{l}\text { Mean pain intensity } \\
P>.09 \text { for younger and } \\
\text { older children }\end{array}$ & - \\
\hline $\begin{array}{l}\text { Stinson et al } \\
(2015)[7]\end{array}$ & $\begin{array}{l}\text { Pearson correla- } \\
\text { tion }\end{array}$ & $\begin{array}{l}\text { Pain intensity } r=0.49- \\
0.63(P<.001) \text {; pain } \\
\text { interference } r=0.53- \\
0.65(P<.001)\end{array}$ & - & - & - \\
\hline
\end{tabular}


Outcome and study Equivalence examination method and results (year)

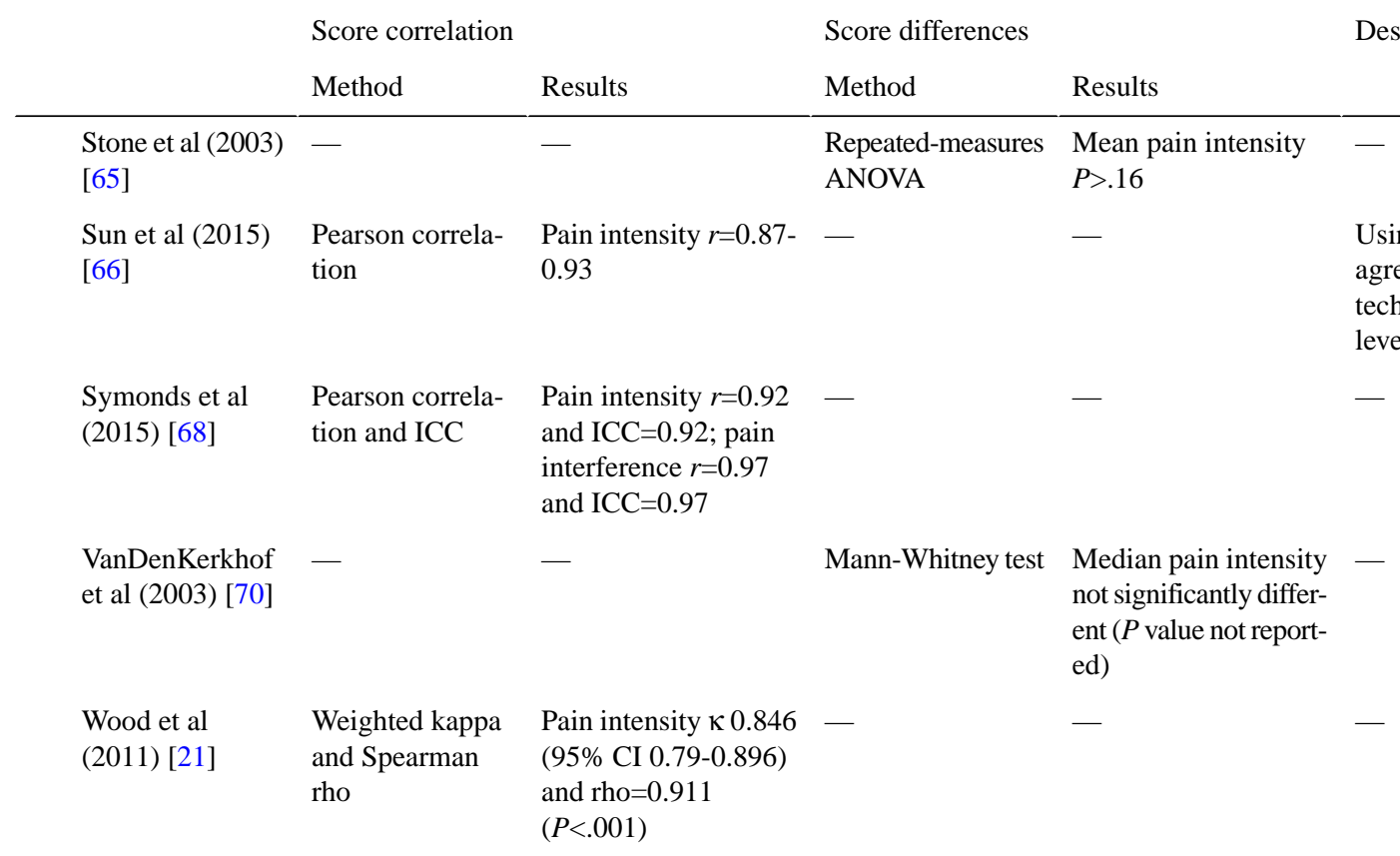

\section{Studies reporting pain score nonequivalence}

Rolfson et al

(2011) [59]

Studies reporting discrepant results

Bedson et al Spearman rho (2019) [28]

Koho et al (2014) ICC

[49]

$\begin{array}{lll}\begin{array}{l}\text { Junker et al } \\ \text { (2008) [46] }\end{array} & - & - \\ & & \\ & & \\ & & \\ \text { Koho et al (2014) } & \text { ICC } & \text { Pain-related fear } \\ {[49]} & & \text { ICC }=0.77(95 \% \text { CI } \\ & & 0.66-0.85)\end{array}$
$\begin{array}{ll}\text { Stinson et al } & \text { Pearson correla- } \\ \text { (2008 and 2014) } & \text { tion and ICC }\end{array}$ $[5,24]$

Stomberg et al (2012) [64]
Mann-Whitney $U$ test

Pain intensity and in- terference baseline paper-based and first 3 days of electronic reports rho $=0.60$ $-0.79(P<.006)$; pain intensity and interference last 3 days of electronic reports and follow-up paper-based rho $=0.40(P<.11)-0.92$ $(P<.001)$

0.66-0.85)

Test not reported

\section{Paired $t$ test}

Pain intensity $r=0.55$ -

0.76 and $\mathrm{ICC}=0.52$ -

$0.75(P<.01)$; pain interference $r=0.77-0.84$ $(P<.01)$

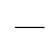

Mantel's test
Descriptive

Using Bland-Altman method, agreement between the data capture techniques shown in the $80 \% \mathrm{CI}$ level.

Mean pain intensity $P=.02$

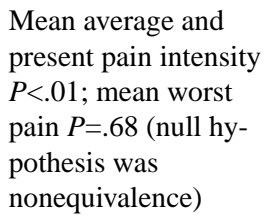

Mean average and present pain intensity $P<.01$; mean worst pain $P=.68$ (null hypothesis was nonequivalence)

Significantly higher mean scores for 2 of 17 scale items using the electronic method ( $P$ value not reported)

Using Bland-Altman method, an agreement between the data capture techniques shown at $95 \% \mathrm{CI}$. 


\begin{tabular}{|c|c|c|c|c|c|}
\hline \multirow{3}{*}{$\begin{array}{l}\text { Outcome and study } \\
\text { (year) }\end{array}$} & \multicolumn{5}{|c|}{ Equivalence examination method and results } \\
\hline & \multicolumn{2}{|l|}{ Score correlation } & \multicolumn{2}{|l|}{ Score differences } & \multirow[t]{2}{*}{ Descriptive } \\
\hline & Method & Results & Method & Results & \\
\hline $\begin{array}{l}\text { Suso-Ribera et al } \\
\text { (2018) [67] }\end{array}$ & $\begin{array}{l}\text { Pearson correla- } \\
\text { tion }\end{array}$ & $\begin{array}{l}\text { Pain intensity and in- } \\
\text { terference } r=0.60-0.81\end{array}$ & $\begin{array}{l}\text { Paired sample } t \\
\text { tests }\end{array}$ & $\begin{array}{l}\text { Averaged weekly pain } \\
\text { interference reports } \\
\text { from app significantly } \\
\text { lower than verbally or } \\
\text { paper-based recalled } \\
\text { interference verbal } \\
\text { over the week } P<.001\end{array}$ & - \\
\hline $\begin{array}{l}\text { Wæhrens et al } \\
\text { (2015) [72] }\end{array}$ & ICC & $\begin{array}{l}\text { Pain intensity and } \\
\text { pain interference } \\
\text { ICC }=0.76-0.98(95 \% \\
\text { CI } 0.50-0.99)\end{array}$ & - & - & - \\
\hline
\end{tabular}

\footnotetext{
${ }^{\mathrm{a}}$ ICC: intraclass correlation coefficient.

${ }^{\mathrm{b}} \mathrm{N} / \mathrm{A}$ : not applicable.

${ }^{\mathrm{c}}$ ANOVA: analysis of variance.

${ }^{\mathrm{d}}$ ANCOVA: analysis of covariance.

${ }^{\mathrm{e}}$ FPS-R: Faces Pain Scale-Revised

fVAS: Visual Analog Scale.

${ }^{g}$ CAS: Color Analogue Scale.

${ }^{\mathrm{h}}$ NRS: Numerical Rating Scale.
}

Across all methods used to compare scores, 82\% (36/44) studies demonstrated equivalence between scores reported electronically or using conventional methods. One of these 44 studies (2\%) reported nonequivalent scores between data collection methods, and 16\% (7/44) studies reported discrepant results. Among studies reporting nonequivalence or discrepancies, purported reasons were recall bias, differences in question layout wherein paper assessments made all items visible to participants simultaneously allowing item scoring in relation to other responses, capacity to change item response using paper methods, and differences in scale presentation (eg, numerical values for NRS not shown using electronic data capture method).

\section{Quantitative Synthesis of Score Equivalence}

A forest plot for correlations for score equivalence between data collection modalities is shown in Figure 3. The weighted summary correlation coefficient was 0.92 (95\% CI 0.88-0.95, $\mathrm{n}=1961)$ and considerable heterogeneity $\left(I^{2}=95 \%\right)$ was observed across studies. Studies using ICC or weighted kappa produced summary correlations that were similar in magnitude to those using Pearson or Spearman rho correlations (ie, 0.91, 95\% CI $0.90-0.92, \mathrm{n}=1360, I^{2}=95 \%$; and $0.85,95 \%$ CI $0.82-0.87$, $\mathrm{n}=1159, I^{2}=95 \%$, respectively). One study met our predefined criterion for extreme effect size [43]. Removing this study from the analysis did not substantially decrease the heterogeneity $\left(I^{2}=94 \%\right)$, and the summary correlation was essentially unchanged at $0.90(95 \%$ CI $0.86 .0 .93, \mathrm{n}=1937)$. Visual inspection of the funnel plot showed asymmetry, suggesting a possible publication bias (Multimedia Appendix 2). 
Figure 3. Summary correlation coefficient for pain intensity and interference data collected via electronic and conventional data capture methods (The $\mathrm{I}^{2}$ and $P$ values for heterogeneity are $95 \%$ and $<0.00001$ respectively; the $\mathrm{Z}$ and $P$ values for the overall effect are 14.4 and $<0.00001$ respectively; POP: population; $\mathrm{R}^{*}$ : correlation coefficient; LCL: lower confidence interval limit; UCL: upper confidence interval limit; WGHT: weight).

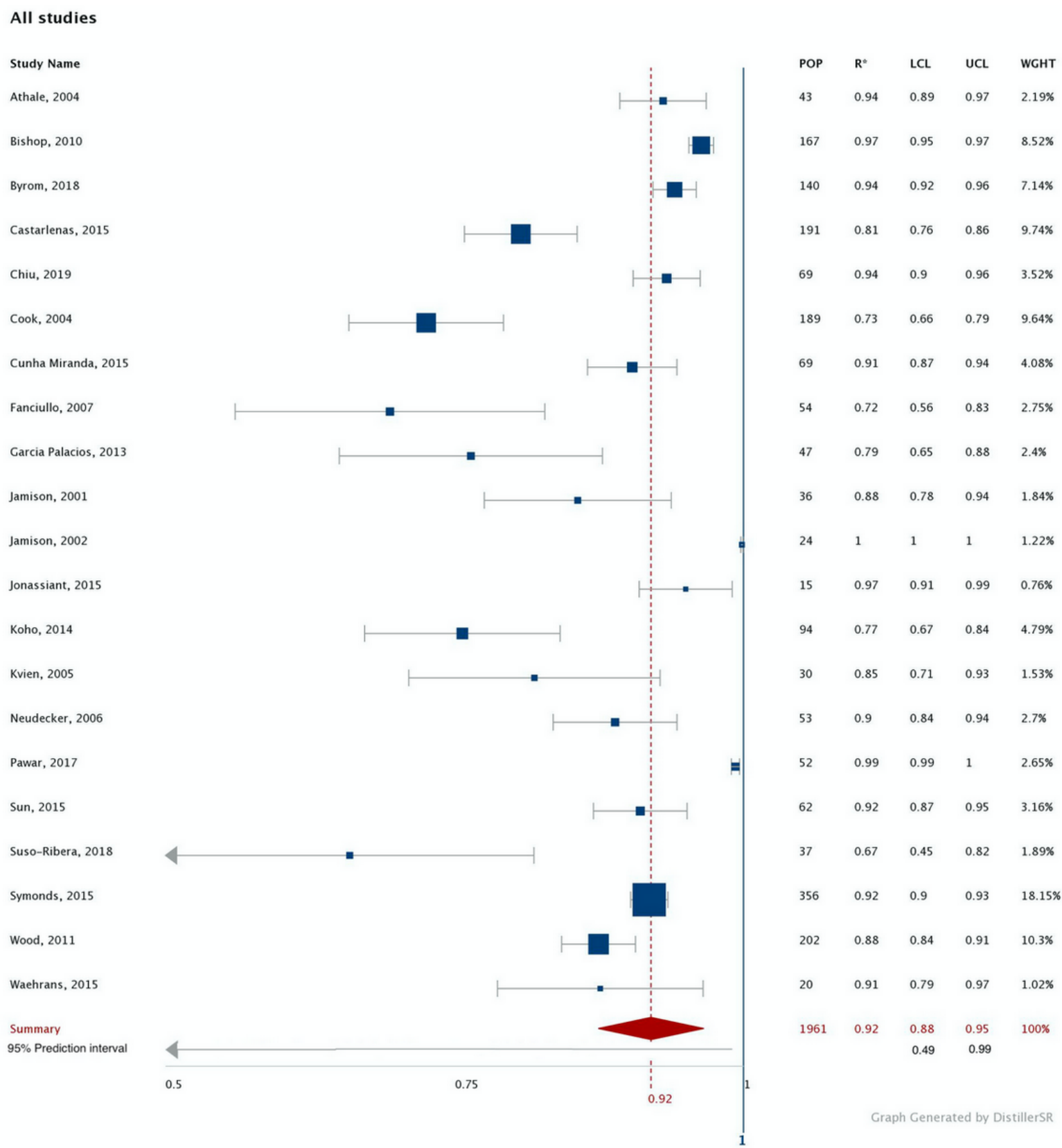

Most studies used the same measure $(n=16)$ versus a different measure $(n=5)$ to assess pain via electronic and conventional modalities, and heterogeneity was high in both subgroups. The summary correlation was 0.93 in studies using the same measure (95\% CI $0.89-0.96, \quad \mathrm{n}=1475, \quad I^{2}=96 \%, 95 \%$ prediction interval $=0.45-0.99$ ) and 0.86 in studies using different measures (95\% CI $0.74-0.93, \mathrm{n}=526, I^{2}=90 \%, 95 \%$ prediction interval -0.01-0.99). In the case of data collection duration, 14 studies collected pain data from participants once and 7 collected data on multiple occasions. The summary correlation was 0.92 in studies that collected pain data once (95\% CI 0.88-0.95, $\mathrm{n}=1678$, $I^{2}=95 \%, 95 \%$ prediction interval $\left.0.57-0.99\right)$ and 0.92 in studies that collected pain data from participants more than once $(95 \%$ CI $0.75 .0 .98, \mathrm{n}=283, I^{2}=96 \%, 95 \%$ prediction interval $-0.61-0.99)$. Heterogeneity remained high despite stratification by the duration of data collection.

\section{Data Completeness}

Overall, 45\% (24/53) studies reported the completeness of data collected via electronic or conventional methods (Table 3). All of these studies compared an electronic data capture modality to paper-based assessments with $8 \%$ (2/24) paper-based assessments being mailed to participants. The assessment of data completeness differed across studies and was largely defined as either the percentage of study participants not 
completing pain assessments or the percentage of missing or incomplete pain assessments. In total, 37\% (9/24) studies reported superior data completeness in the electronic data capture group, $33 \%(8 / 24)$ studies reported superior data completeness in the conventional data capture group, $8 \%(2 / 24)$ studies reported mixed results, and 20\% (5/24) studies did not conduct a direct comparison between data collection modalities, but reported a high data completeness using electronic data capture. 
Table 3. Summary of study results related to data completeness.

\begin{tabular}{lll}
\hline Authors (year) & $\begin{array}{l}\text { Electronic data collection } \\
\text { modality }\end{array}$ & $\begin{array}{l}\text { Conventional data collection } \\
\text { modality }\end{array}$ \\
\hline Allena et al (2012) [25] & Complete records: 98\% & Not reported \\
Athale et al (2004) [26] & Missing data: 7/63 (11\%) & $\begin{array}{l}\text { Missing data: } 16 / 63(25 \%) \\
\text { Bandarian-Balooch et al (2017) }\end{array}$ \\
[27] & & $\begin{array}{l}\text { Long-paper diaries had signifi- } \\
\text { cantly higher missing data } \\
\text { scores in data completion than } \\
\text { the e-diaries and short-paper } \\
\text { diaries }(P<.05) \text {. The short-paper } \\
\text { diary had significantly more } \\
\text { missing data than the mobile } \\
\text { phone groups }(P<.05) \text { but was } \\
\text { not significantly different than } \\
\text { the computer group. }\end{array}$ \\
& & \\
& &
\end{tabular}

Bedson et al (2019) [28] Recordings were made on Not reported $73.3 \%$ of days

Bishop et al (2010) [29]

Christie et al (2014) [33]

Gaertner et al (2004) [38]

Garcia-Palacios et al (2013) [39] Complete records: 18.2

Heiberg et al (2007) [40]

Jamison et al (2001) [15]

Junker et al (2008) [46]

Khan et al (2019) [47]

Marceau et al (2007) [52]

Palermo et al (2004) [56]

Ritter et al (2004) [58]

Rolfson et al (2011) [59]

Stinson et al (2008 and 2014) $[5,24]$
Missing data: $8 \%$ of all daily assessments $(86.66 \%)$

Not reported

Missing data: 15 responses ( $0.004 \%$ of items)

Response rate: $97.9 \%$

Median value for missing daily data entries: 1 for both periods

Compliance with reporting: $89.9 \%$

Mean number of queries: 1.53 (2.70)

Complete records: $397 / 461$ $(86.1 \%)$

Compliance: $83.3 \%$

Response rate: $87.5 \%$

Response rate: $49 \%$

Response rate: $78 \%$ and $73 \%$ for 2- and 3-week study protocols, respectively
Not reported time) $55.9 \%$ $0.90(0.87)$ $(100 \%)$
Definitions

Defined as the percent of participants completing all assessments

Defined as the percent of participants completing assessments

Defined as the number of missing items irrespective of inaccurate completion

Defined as percentage of days on which participants recorded data

Missing data: 3 responses $(0.0007 \%$ of items $)$

Defined as the total number of missed assessment items across all participants

Defined as the percent of possible text message-based pain assessments completed cross all participants

Missing data: 0\% (participants reported retrospectively completing assessments when they forgot to do so at the scheduled

Complete records: 11.1 $(52.95 \% ; P<.01)$

Median value for missing daily data entries: 0 for both periods

Compliance with reporting:

Defined as the percent assessments not completed across all participants over 14 days

Defined as mean number of complete assessments across participants out of possible records

Defined as median number of missing assessments over 21 days

Defined as percent of assessments completed each day for 1 year (365 days; electronic assessments) and percent of assessments completed for 7 days each month for 1 year (84 days; conventional assessment)

Defined as number of missing items across each assessment

Noticeably more missing on the conventional method when compared with the electronic pain assessment

Mean (SD) number of queries:

Complete records: 583/583

Compliance: $46.7 \%(P<.001)$

Defined as concerns about a specific data point raised by the data manager or study coordinator relating to inappropriate or missing data

Defined as the number of assessments completed across all participants

Defined as the percent of assessments completed over the 7 days

Response rate: $83.1 \%(P=.19) \quad$ Defined as percent of participants who completed assessments

Response rate: $92 \%(P<.01) \quad$ Defined as percent of participants who completed assessments

Response rate: $93 \%$ in week 1 Defined as $100 \%$ when 3 diary entries were and $92 \%$ in week 2 (not report- completed for each of the 14 or 21 days of data ed for 3-week protocol) collection 


\begin{tabular}{|c|c|c|c|}
\hline Authors (year) & $\begin{array}{l}\text { Electronic data collection } \\
\text { modality }\end{array}$ & $\begin{array}{l}\text { Conventional data collection } \\
\text { modality }\end{array}$ & Definitions \\
\hline Stinson et al (2012) [63] & $\begin{array}{l}\text { Missing data using Mobile } \\
\text { phones: } 5.26 \% \text { (younger chil- } \\
\text { dren), } 3.42 \% \text { (older children); } \\
\text { missing data using computer: } \\
0 \% \text { (younger children), } 0.14 \% \\
\text { (older children) }\end{array}$ & $\begin{array}{l}\text { Missing data: } 0 \% \text { (younger } \\
\text { children), } 1.16 \% / 77 \text { (older } \\
\text { children; } P=.047 \text { ) }\end{array}$ & $\begin{array}{l}\text { Defined as the percent of assessment items not } \\
\text { answered by participants }\end{array}$ \\
\hline Stinson et al (2015) [7] & $\begin{array}{l}\text { Response rate: } 72.2 \% \text { and } \\
47.1 \% \text { for } 2 \text { - and } 3 \text {-week study } \\
\text { protocols, respectively }\end{array}$ & Not reported & $\begin{array}{l}\text { Defined as } 100 \% \text { when participants completed } \\
2 \text { diary entries per day for } 14 \text { days }\end{array}$ \\
\hline Stomberg et al (2012) [64] & $\begin{array}{l}\text { Response rate on the day of } \\
\text { surgery: } 35 \% \text {; response rate on } \\
\text { days } 2-4 \text { postoperatively: } \\
100 \% \text {; response rate on days } 5 \text { - } \\
6 \text { postoperatively: } 69 \%\end{array}$ & $\begin{array}{l}\text { Response rate on the day of } \\
\text { surgery: } 41 \% \text {; response rate on } \\
\text { days } 2-4 \text { postoperatively: } \\
100 \% \text {; not required to complete } \\
\text { questionnaire on days } 5-6\end{array}$ & $\begin{array}{l}\text { Defined as the percent of participants completing } \\
\text { assessments }\end{array}$ \\
\hline Stone et al (2003) [65] & $\begin{array}{l}\text { Response rate } 3 \text { prompts per } \\
\text { day: } 93.5 \% \text {; response rate } 6 \\
\text { prompts per day: } 93.9 \% \text {; re- } \\
\text { sponse rate } 12 \text { per day } 95.5 \%\end{array}$ & Response rate: $100.0 \%$ & $\begin{array}{l}\text { Defined as the percent of participants completing } \\
\text { assessments }\end{array}$ \\
\hline Suso-Ribera et al (2018) [67] & Response rate: $75.7 \%$ & Not reported & $\begin{array}{l}\text { Defined as the percent of completed assessments } \\
\text { out of all possible assessments }\end{array}$ \\
\hline VanDenKerkhof et al (2003) [70] & $\begin{array}{l}\text { NRS }^{b} \text { score documentation } \\
\text { rate: } 100 \%\end{array}$ & $\begin{array}{l}\text { NRS score documentation rate: } \\
90-97 \%\end{array}$ & $\begin{array}{l}\text { Defined as the percentage of time an NRS score } \\
\text { was documented during a patient encounter }\end{array}$ \\
\hline VanDenKerkhof et al (2004) [71] & $\begin{array}{l}\text { Complete records pain scores: } \\
64.7 \% \text {; complete records nau- } \\
\text { sea, pruritis and sedation side } \\
\text { effects: } 100 \% \text {; complete } \\
\text { records hypotension side effect: } \\
20.6 \%\end{array}$ & $\begin{array}{l}\text { Complete records pain scores: } \\
43.6 \%(P=.07) \text {; complete } \\
\text { records nausea, pruritis and se- } \\
\text { dation side effects: } 12.8-33.3 \% \\
\text { of paper assessments } \\
(P=<.001) \text {; complete records } \\
\text { hypotension side effect: } 5.1 \% \\
(P=.07)\end{array}$ & $\begin{array}{l}\text { Percent of assessments where outcome was } \\
\text { recorded }\end{array}$ \\
\hline
\end{tabular}

${ }^{\mathrm{a}} \mathrm{N} / \mathrm{A}$ : not applicable.

${ }^{\mathrm{b}}$ NRS: Numerical Rating Scale.

\section{Ease of Use}

The ease of use of electronic and/or conventional pain data capture methods was reported in 45\% (24/53) studies (Table 4). Ease was assessed subjectively using administered quantitative or qualitative surveys or verbal reports in all studies. Overall, electronic data collection modalities were considered easy to use by patients in pain or their care providers. In $91 \%$
(22/24) of the studies, the electronic modality was considered easy to use, easy to understand, or easy to review or report pain. In all, 29\% (7/24) studies conducted inferential testing comparing ease between pain data capture modalities. Of these studies, $57 \%$ (4/7) showed that electronic versions were significantly easier to use, $14 \%$ (1/7) study showed that the paper version was significantly easier to use, and 28\% (2/7) studies showed no significant differences between groups. 
Table 4. Summary of study results related to ease of use.

\begin{tabular}{llll}
\hline Study (year) & Electronic data collection modality & $\begin{array}{l}\text { Conventional data collection } \\
\text { modality }\end{array}$ & Conclusion \\
\hline Allena et al & Easy to understand: mean 8.7/10; easy to use: & $\begin{array}{l}\text { Easy to understand: mean } \\
8.3 / 10 ; \text { easy to use: mean }\end{array}$ & Electronic format significantly $(P<.01)$ easier. \\
$(2012)[25]$ & mean $8.9 / 10$ & $7.9 / 10$ & \\
& & $5 / 19(26 \%)$ rated paper as easi- & Not reported \\
Athale et al & $9 / 19(47 \%)$ rated computer as easier & er &
\end{tabular}

Bandarian-

Balooch et al

(2017) [27]

Bedson et al

(2019) [28]

Bishop et al (2010) [29]

Ease of use (all electronic methods combined) mean $6.58 / 10$

$100 \%$ reported easy to read

17 comments on easy completion

Blum et al (2014) $79 \%$ reported no difficulty with using electronic [30] method

Cook et al $2004 \quad 39 \%$ of patients stated easier to understand and [34] complete

Freynhagen et al No issues with the use of the PDA ${ }^{\mathrm{a}}$ (2006) [37]

Gaertner et al (2004) [38]

Garcia-Palacios et al (2013) [39]

Jaatun et al

(2014) [42]

[49]

$54 \%$ found more complicated

$15 / 40(37 \%)$ rated easier to use

Both physicians found electronic pain reports easier to read and evaluate than the paper maps. rated difficult to complete

MacKenzie et al $\quad 54 / 63(85.7 \%)$ rated easy to complete (2011) [51]

Marceau et al (2007) [52]

$32 / 36(89 \%)$ rated easy to understand and use; $30 / 36(83 \%)$ rated easy to record data

Marceau et al (2010) [53]

Palermo et al (2004) [56]

Pawar et al (2017) [57]

Serif et al (2005) Some users, especially those with arthritis and/or [62]

Stinson et al

(2008 and 2014)

$[5,24]$

Stinson et al

(2012) [63]

Stinson et al

(2015) [7]

Stomberg et al (2012) [64] poorer eyesight encountered difficulties in using the electronic modality, but ease of use was general consensus

Majority found the electronic format easy to use

$29 / 43(67.4 \%)$ rated easy to use and understand

$15 / 18(83 \%)$ rated easy or very easy to remember to fill out

$70.58 \%$ rated as easy to use be easier to understand than the handheld device

$94.6 \%$ and $91.7 \%$ of participants in the 2- and 3- Not reported week studies, respectively, found electronic diary interfered only minimally with activities

Mean difficulty in using electronic modality: $1.31 / 10$

Not reported

Not reported

Not reported

Not reported scribed
Ease of use: mean $6.17 / 10$

Not reported

16 comments on easy completion

Not reported

$24 \%$ of patients stated easier to understand and complete

Not reported

Not reported

$42 \%$ found more complicated

$4 / 40(10 \%)$ rated easier to use

Not reported

$63 / 93(68 \%)$ rated easy to complete, 10/93 (11\%) rated difficult to complete

Not reported

$27 / 36(75 \%)$ rated easy to understand and use; $3 / 36(8 \%)$ rated easy to record data

$32 / 35(91.4 \%)$ rated easy to use and understand

$8 / 15(53 \%)$ rated easy or very easy to remember to fill out

No difficulties with use dearies

Not reported

Not reported

Not reported

Not reported

Not reported

Not reported

Not reported

Not reported

Not reported

Not reported

Not reported ease of use

Not reported

Not reported
The long-paper diary was rated as significantly $(P<.02)$ less easy to use than the other di-

No significant difference between modalities

No significant difference in ease of understanding and use. Significantly $(P<.001)$ higher ease of recording data rating for electronic modality.

Significantly $(P=.01)$ higher ease of use and understanding for paper modality.

No significant difference between modalities

Significant difference $(P=.03)$ in opinion of

J Med Internet Res 2020 | vol. 22 | iss. 6 | e16480 | p. 24 (page number not for citation purposes) 


\begin{tabular}{llll}
\hline Study (year) & Electronic data collection modality & $\begin{array}{l}\text { Conventional data collection } \\
\text { modality }\end{array}$ & Conclusion \\
\hline $\begin{array}{l}\text { Suso-Ribera et al } \\
(2018) \text { [67] }\end{array}$ & $\begin{array}{l}\text { 100\% of participants found the app extremely } \\
\text { easy to use }\end{array}$ & Not reported & Not reported \\
$\begin{array}{l}\text { Wæhrens et al } \\
(2015) \text { [72] }\end{array}$ & Not reported & None found paper easier to use & Not reported \\
\hline
\end{tabular}

${ }^{\mathrm{a} P D A}$ : personal digital assistant.

\section{Efficiency}

In total, $30 \%(16 / 53)$ studies reported on the time to complete pain assessments (Table 5). In all, 44\% (7/16) of these studies provided some evidence that pain assessments completed via the electronic modality were quick to complete; $19 \%(3 / 16)$ of these studies provided some evidence that conventional methods to assess pain were quicker; and 1 of 16 studies $(6 \%)$ showed mixed results where differences in between-assessment modality completion times differed by participant group (eg, older children, parents, and younger children). In all, 25\% (4/16) studies indicated that there were no differences in time to complete assessments across methods. Overall, in studies that directly measured the time to complete pain assessments $[28,50,51,57,62,63,70,71]$, the difference in mean times to complete assessments was minimal (ie, <5.6 min). 
Table 5. Summary of study results related to efficiency.

\begin{tabular}{|c|c|c|c|}
\hline Study & Electronic data collection modality & $\begin{array}{l}\text { Conventional data collection } \\
\text { modality }\end{array}$ & Study author conclusions \\
\hline $\begin{array}{l}\text { Bedson et al (2019) } \\
{[28]}\end{array}$ & $\begin{array}{l}\text { Mean and max times to complete pain assessment: } 2 \\
\text { and } 5 \text { min }\end{array}$ & Not reported & Not reported \\
\hline $\begin{array}{l}\text { Bishop et al (2010) } \\
\text { [29] }\end{array}$ & 19 comments on quick to complete & $\begin{array}{l}9 \text { comments on quick to com- } \\
\text { plete }\end{array}$ & Not reported \\
\hline $\begin{array}{l}\text { Blum et al (2014) } \\
\text { [30] }\end{array}$ & $70 \%$ completed pain assessment in under $5 \mathrm{~min}$ & $\begin{array}{l}88 \% \text { completed pain assessment } \\
\text { in under } 5 \text { min (questionnaire } \\
\text { had fewer times than electronic } \\
\text { modality) }\end{array}$ & Not reported \\
\hline $\begin{array}{l}\text { Gaertner et al (2004) } \\
\text { [38] }\end{array}$ & $\begin{array}{l}\text { No difference in time to complete pain assessments } \\
\text { between groups (always less than } 15 \mathrm{~min} / \text { day) }\end{array}$ & $-^{\mathrm{a}}$ & Not reported \\
\hline $\begin{array}{l}\text { Heiberg et al (2007) } \\
{[40]}\end{array}$ & $\begin{array}{l}\text { Time to complete the pain assessment similar between } \\
\text { groups }\end{array}$ & - & Not reported \\
\hline $\begin{array}{l}\text { Kim et al (2016) } \\
{[48]}\end{array}$ & $\begin{array}{l}68.7 \% \text { responded that the time to complete pain assess- } \\
\text { ments positive or very positive }\end{array}$ & Not reported & $\begin{array}{l}\text { Significant relationship regarding } \\
\text { participants evaluation of the time } \\
\text { to complete electronic questionnaire } \\
P<.001\end{array}$ \\
\hline $\begin{array}{l}\text { Kvien et al (2005) } \\
{[50]}\end{array}$ & $\begin{array}{l}\text { Mean (SD) time to complete pain assessment: } 30.5 \\
(16.0) \mathrm{min}\end{array}$ & $\begin{array}{l}\text { Mean (SD) time to complete pain } \\
\text { assessment: } 24.9(27.0) \mathrm{min}\end{array}$ & $\begin{array}{l}\text { No significant difference between } \\
\text { groups }(P=.11)\end{array}$ \\
\hline $\begin{array}{l}\text { MacKenzie et al } \\
(2011)[51]\end{array}$ & $\begin{array}{l}\text { Mean time to complete pain assessment: } 25.0 \mathrm{~min} \\
\text { (range } 5 \text { to } 80 \mathrm{~min} \text { ) }\end{array}$ & $\begin{array}{l}\text { Mean time to complete pain as- } \\
\text { sessment: } 24.2 \mathrm{~min} \text { (range } 5 \text { to } \\
60 \mathrm{~min} \text { ) }\end{array}$ & Not reported \\
\hline $\begin{array}{l}\text { Pawar et al (2017) } \\
\text { [57] }\end{array}$ & $\begin{array}{l}\text { Mean time to complete pain assessment: } 1.28 \mathrm{~min} \\
\text { (range } 0.83-2.63 \mathrm{~min} \text { ) }\end{array}$ & $\begin{array}{l}\text { Mean time to complete pain as- } \\
\text { sessment: } 3.7 \text { min (range } 2.42- \\
5.23 \mathrm{~min} \text { ) }\end{array}$ & Not reported \\
\hline $\begin{array}{l}\text { Serif et al (2005) } \\
{[62]}\end{array}$ & Mean time to complete pain assessment: 47 seconds & $\begin{array}{l}\text { Mean time to complete pain as- } \\
\text { sessment: } 267 \text { seconds }\end{array}$ & Not reported \\
\hline $\begin{array}{l}\text { Stinson et al }(2008 \\
\text { and 2014) }[5,24]\end{array}$ & Most adolescents found the app quick to complete & Not reported & Not reported \\
\hline $\begin{array}{l}\text { Stinson et al (2012) } \\
\text { [63] }\end{array}$ & $\begin{array}{l}\text { Computer: mean (SD) time to complete pain assess- } \\
\text { ment: } 3.40 \text { (1.53) min for older children, } 4.00(1.71) \\
\text { min for parents and } 1.64(1.50) \text { min for younger chil- } \\
\text { dren; Mobile phone: mean (SD) time to complete pain } \\
\text { assessment: } 5.90(2.79) \text { min for older children, } 7.00 \\
\text { (4.08) min for parents and } 1.82(1.17) \text { min for younger } \\
\text { children }\end{array}$ & $\begin{array}{l}\text { Mean (SD) time to complete pain } \\
\text { assessment: } 3.08(1.66) \mathrm{min} \text { for } \\
\text { older children, } 2.28(1.32) \mathrm{min} \\
\text { for parents and } 1.91(1.81) \mathrm{min} \\
\text { for younger children }\end{array}$ & $\begin{array}{l}\text { Completion times significantly } \\
\text { longer in electronic group for older } \\
\text { children and parents }(P=.001) \text {. No } \\
\text { significant difference for younger } \\
\text { children }(P=.64) \text { who completed a } \\
\text { shorter assessment. }\end{array}$ \\
\hline $\begin{array}{l}\text { Stinson et al (2015) } \\
\text { [7] }\end{array}$ & $\begin{array}{l}93.2 \% \text { and } 91.7 \% \text { of participants in the } 2 \text { - and } 3 \text {-week } \\
\text { studies, respectively, found electronic diary quick to } \\
\text { complete }\end{array}$ & Not reported & Not reported \\
\hline $\begin{array}{l}\text { Stomberg et al } \\
\text { (2012) [64] }\end{array}$ & $\begin{array}{l}\text { Participants reported electronic modality not time } \\
\text { consuming }\end{array}$ & Not reported & Not reported \\
\hline $\begin{array}{l}\text { VanDenKerkhof et } \\
\text { al (2003) [70] }\end{array}$ & $\begin{array}{l}\text { Median (IQR) time to complete pain assessment: } 206 \\
\text { (70) seconds }\end{array}$ & $\begin{array}{l}\text { Median (IQR) time to complete } \\
\text { pain assessment: } 153(85) \text { sec- } \\
\text { onds }\end{array}$ & $\begin{array}{l}\text { Completion time significantly } \\
\text { longer time to complete using elec- } \\
\text { tronic modality }(P<.001)\end{array}$ \\
\hline $\begin{array}{l}\text { VanDenKerkhof et } \\
\text { al (2004) [71] }\end{array}$ & $\begin{array}{l}\text { Median (IQR) time to complete pain assessment } 2.8 \\
\text { min }\end{array}$ & $\begin{array}{l}\text { Median (IQR) time to complete } \\
\text { pain assessment } 2.7 \mathrm{~min}\end{array}$ & $\begin{array}{l}\text { No significant difference between } \\
\text { groups }(P=.74)\end{array}$ \\
\hline
\end{tabular}

${ }^{\mathrm{a}} \mathrm{N} / \mathrm{A}$ : not applicable.

\section{Acceptability}

Data related to the comparative acceptability of each pain assessment modality were collected in $60 \%(32 / 53)$ studies [5,7,21,22,24-27,29,30,34,36,38-42,47-53,56,57,60,61,63,64,66,69,72]. Overall, electronic programs to assess pain are highly acceptable to patients. In total, $19(83 \%)$ of the 23 studies
$[21,22,25,26,30,34,36,38-42,49-51,57,60,72,73]$ that directly surveyed patients reported that the electronic format was the preferred data collection method, compared with 1 of 23 studies (4\%) [69], which reported that the conventional data collection method was preferable. This study indicated that age was related to patient preference, with younger patients (mean age 45 years) tending to prefer the internet and older patients (mean age 54 
years), preferring the telephone-based data collection; 9\% (2/23) studies reported discrepant results [66]. One of these studies reported that children aged $<8$ years favored the electronic assessment method, whereas the parents of these children and children aged 8 to 18 years had no preference. The other study reported that the preferred modality differed depending on the type of pain measurement instrument used. One study (4\%) found no difference in participant satisfaction between electronic and conventional pain instruments [47]. Nine studies did not ask patients to specifically declare a preference for assessment modality but still reported high patient satisfaction with the electronic method [5,7,27,29,48,52,53,56,64,74].

\section{Discussion}

\section{Principal Findings}

This is the first systematic review and meta-analysis to compare electronic and conventional data collection methods for pain-related outcomes. The results from our review suggest strong correspondence in pain scores collected across electronic and conventional modalities as well as ease of use and acceptability for electronic data capture methods. Comparisons of data completeness and efficiency showed mixed results in terms of the superiority of electronic modalities over conventional methods. Overall, these results indicate that electronic data capture is a viable means to assess pain and has the potential to overcome many of the known limitations associated with conventional methods.

The capacity to obtain equivalently scored data from patients across electronic and conventional data capture modalities is paramount to the use of more novel collection methods in clinical and research settings. Studies included in this review (ie, in $82 \%$ of cases) commonly reported on the correspondence of pain scores between assessments. Regardless of whether the data analyses were qualitative or quantitative, the general consensus across studies was that pain was reported equivalently across assessment modalities. The meta-analysis of correlations between scores reported electronically and conventionally resulted in a summary coefficient of 0.92 , indicating high correspondence. The summary coefficients produced by studies reporting ICC or weighted kappa and studies reporting Pearson or Spearman rho coefficients were not different from the overall summary score, suggesting negligible change in patient-reported scores across modalities. These findings agree with those of a meta-analysis published in 2008 that evaluated the equivalence of scores for patient-reported outcomes (not specifically pain) completed using PDA, computer, or tablet and paper-based methods and that showed a summary correlation of 0.90 [9]. Together, these reviews suggest that score equivalence between electronic and conventional data capture methods is a robust finding across patient-reported outcomes.

Despite our use of random effects models, we observed substantial heterogeneity across studies included in the meta-analysis that was not accounted for by the single study that met our criterion for extreme effect size, sensitivity analyses by correlation type, the similarity of pain assessment measure used in each modality, or duration of data collection. Studies varied in terms of study design, participant group, type of electronic and conventional data collection method, and pain measurement instrument — the heterogeneity may be explained by these differences in methodology. For instance, the type of electronic device used to collect pain data varied across studies, meaning that aspects of the device such as interface design, user familiarity, and screen size could each have contributed to our heterogeneous results [11]. The included studies also varied in terms of the type of pain intensity scale or pain interference instrument used (eg, NRS, VAS, etc). Although good congruence in patient self-report across instruments has been shown [75], and that the transfer of the assessment instrument to the electronic format generally appeared to be in good faith, as reported previously, differences in pain ratings across instruments are possible [76]. Irrespective of the observed heterogeneity, the correlation coefficients were strong across all studies with no reported coefficients less than 0.64 , suggesting that heterogeneity should not temper the meta-analysis conclusion.

The collection of high-quality and complete patient-reported data is of utmost importance to clinicians, researchers, and study sponsors [12]. Data completeness was a commonly reported comparison outcome across data collection methods in the included studies. The results regarding the superiority of data completeness were mixed. However, the electronic method was most often associated with more complete data being collected. Ultimately, methodological and logistical issues related to paper-based data collection methods may support the use of electronic data capture. For instance, research has shown that the completeness and accuracy of pain data collected via paper methods is adversely impacted by patients back-filling diaries and, therefore, introducing recall bias into datasets (a behavior that can be rendered impossible using electronic methods) [8]. In addition, the capacity to efficiently and cost-effectively develop large databases for clinical and research purposes may be improved with electronic data capture. For instance, one of the studies included in this review [47] showed that over 4-fold more research assistant time was required to manage postoperative pain data collected using conventional means compared with electronic data. This finding suggests that cost savings may result from the use of electronic pain assessments in research, and this savings might be pronounced at scale. Furthermore, the likelihood of inaccurate or missing data in these databases resulting from human input error is reduced in the case of electronic entry [77].

Almost all studies that assessed ease indicated, in some manner, that electronic methods were easy to use, easy to understand, or easy to review or report pain. The time difference required to complete pain assessments via each data collection method was minimal, and the majority of studies showed that the electronic method required equal or less time to complete than conventional methods. The methodological advantages of electronic data capture include high-density sampling in all environments. Evidence of ease of use and efficiency in electronic data capture is useful to researchers and clinicians considering leveraging these utilities to collect repeated ecologically relevant pain assessments [78].

Electronic data capture was also shown to be a highly acceptable method for pain assessment and was more likely to be the 
method of choice for reporting by patients. These findings agree with those of previous studies comparing electronic and conventional methods [10]. Given the heterogeneity of electronic pain data capture methods, participant populations, and sampling densities of included studies, our results suggest acceptability across a range of data collection contexts. This result is meaningful as the acceptability of an intervention has been linked to adoption, especially in relation to long-term sustainability [79].

\section{Limitations}

Some included studies did not administer the same pain measurement instrument or use the same sampling schedule via electronic and conventional methods, making it difficult to directly compare results across modalities. Owing to variations in study design and the fact that our outcomes of interest were often times not the main objective of our included studies, we did not perform an assessment of quality for included studies; instead, we elected to include all identified studies in our review. Our results and conclusions are, therefore, the product of studies that may have included significant methodological weaknesses. In addition, as is an issue with all systematic reviews, we are constrained by possible publication bias, which was suggested by the funnel plot inspection of our quantitative synthesis data. However, given the objectives of the studies we included, we believe that the likelihood of a file-drawer effect is low. Finally, we included studies conducted in controlled (eg, research and health care institutions) and uncontrolled (eg, participant home) environments. We are, therefore, limited in our ability to make more definitive conclusions about our outcomes as they pertain to ecologically relevant data collection, which is considered a major methodological advantage of the electronic method.

\section{Conclusions}

Overall, this review demonstrates that electronic pain-related data capture methods are comparable with conventional methods in terms of score equivalence, data completeness, ease, efficiency, and acceptability. Specifically, pain-related outcome scores reported across methods were congruent in terms of score correlations and mean or median differences between scores. Data completeness, ease of use, efficiency, and acceptability outcomes were also comparable or superior using electronic data capture. Our results suggest that electronic methods are a feasible means to collect pain data, and the use of these methods is likely to increase with the ubiquitous use of mobile phones outside of the clinical or research setting. However, a critical caveat to this conclusion relates to the validation of pain instruments that are implemented electronically. To ensure the collection of accurate data, rigorous methods should be used to establish the sound psychometric properties of electronic pain measurement instruments. Validation of electronic methods will facilitate the capture of pain data in clinical settings but will also support its use in data collection for interventional research, an area that has largely not been explored to date [6].

\section{Conflicts of Interest}

PS works for and owns shares of a digital health company that makes electronic medical records. All other authors have no conflicts of interest to disclose.

\section{Multimedia Appendix 1}

Sample search strategy.

[DOCX File, 12 KB-Multimedia Appendix 1]

\section{Multimedia Appendix 2}

Funnel plot of 21 studies presenting correlations for score equivalence between electronic and conventional pain data collection modalities.

[PNG File, 48 KB-Multimedia Appendix 2]

\section{References}

1. Melzack R. Evolution of the neuromatrix theory of pain. The Prithvi Raj lecture: presented at the third world congress of world institute of pain, Barcelona 2004. Pain Pract 2005 Jun;5(2):85-94. [doi: 10.1111/j.1533-2500.2005.05203.x] [Medline: 17177754]

2. Schiavenato M, Craig KD. Pain assessment as a social transaction: beyond the 'gold standard'. Clin J Pain 2010 Oct;26(8):667-676. [doi: 10.1097/AJP.0b013e3181e72507] [Medline: 20664341]

3. Asghari A, Nicholas MK. Pain self-efficacy beliefs and pain behaviour. A prospective study. Pain 2001 Oct;94(1):85-100. [doi: 10.1016/s0304-3959(01)00344-x] [Medline: 11576748]

4. Schneider S, Stone AA, Schwartz JE, Broderick JE. Peak and end effects in patients' daily recall of pain and fatigue: a within-subjects analysis. J Pain 2011 Mar;12(2):228-235 [FREE Full text] [doi: 10.1016/j.jpain.2010.07.001] [Medline: 20817615]

5. Stinson JN, Jibb LA, Lalloo C, Feldman BM, McGrath PJ, Petroz GC, et al. Comparison of average weekly pain using recalled paper and momentary assessment electronic diary reports in children with arthritis. Clin J Pain 2014 Dec;30(12):1044-1050. [doi: 10.1097/AJP.0000000000000072] [Medline: 24535055] 
6. May M, Junghaenel DU, Ono M, Stone AA, Schneider S. Ecological momentary assessment methodology in chronic pain research: a systematic review. J Pain 2018 Jul;19(7):699-716 [FREE Full text] [doi: 10.1016/j.jpain.2018.01.006] [Medline: 29371113]

7. Stinson JN, Jibb LA, Nguyen C, Nathan PC, Maloney AM, Dupuis LL, et al. Construct validity and reliability of a real-time multidimensional smartphone app to assess pain in children and adolescents with cancer. Pain 2015 Dec;156(12):2607-2615. [doi: 10.1097/j.pain.0000000000000385] [Medline: 26580680]

8. Stone AA, Shiffman S, Schwartz JE, Broderick JE, Hufford MR. Patient non-compliance with paper diaries. Br Med J 2002 May 18;324(7347):1193-1194 [ [FREE Full text] [doi: 10.1136/bmj.324.7347.1193] [Medline: 12016186 ]

9. Gwaltney CJ, Shields AL, Shiffman S. Equivalence of electronic and paper-and-pencil administration of patient-reported outcome measures: a meta-analytic review. Value Health 2008;11(2):322-333 [FREE Full text] [doi:

10.1111/j.1524-4733.2007.00231.x] [Medline: 18380645]

10. Lane SJ, Heddle NM, Arnold E, Walker I. A review of randomized controlled trials comparing the effectiveness of hand held computers with paper methods for data collection. BMC Med Inform Decis Mak 2006 May 31;6:23 [FREE Full text] [doi: 10.1186/1472-6947-6-23] [Medline: 16737535]

11. Belisario JS, Jamsek J, Huckvale K, O'Donoghue J, Morrison CP, Car J. Comparison of self-administered survey questionnaire responses collected using mobile apps versus other methods. Cochrane Database Syst Rev 2015 Jul 27(7):MR000042. [doi: 10.1002/14651858.MR000042.pub2] [Medline: 26212714]

12. Zbrozek A, Hebert J, Gogates G, Thorell R, Dell C, Molsen E, et al. Validation of electronic systems to collect patient-reported outcome (PRO) data-recommendations for clinical trial teams: report of the ISPOR ePRO systems validation good research practices task force. Value Health 2013 Jun;16(4):480-489 [FREE Full text] [doi: 10.1016/j.jval.2013.04.002] [Medline: $\underline{23796281]}$

13. von Korff M, Scher AI, Helmick C, Carter-Pokras O, Dodick DW, Goulet J, et al. United States national pain strategy for population research: concepts, definitions, and pilot data. J Pain 2016 Oct;17(10):1068-1080. [doi:

10.1016/j.jpain.2016.06.009] [Medline: 27377620]

14. Dillon DG, Pirie F, Rice S, Pomilla C, Sandhu MS, Motala AA, African Partnership for Chronic Disease Research (APCDR). Open-source electronic data capture system offered increased accuracy and cost-effectiveness compared with paper methods in Africa. J Clin Epidemiol 2014 Dec;67(12):1358-1363 [FREE Full text] [doi: 10.1016/j.jclinepi.2014.06.012] [Medline: 25135245]

15. Jamison RN, Raymond SA, Levine JG, Slawsby EA, Nedeljkovic SS, Katz NP. Electronic diaries for monitoring chronic pain: 1-year validation study. Pain 2001 Apr;91(3):277-285. [doi: 10.1016/s0304-3959(00)00450-4] [Medline: 11275385]

16. Canadian Internet Registration Authority. 2018. Canada's Internet Factbook 2019 URL: https://www.cira.ca/resources/ corporate/factbook/canadas-internet-factbook-2019 [accessed 2019-10-09]

17. Moher D, Liberati A, Tetzlaff J, Altman DG, PRISMA Group. Preferred reporting items for systematic reviews and meta-analyses: the PRISMA statement. Ann Intern Med 2009 Aug 18;151(4):264-9, W64. [doi:

10.7326/0003-4819-151-4-200908180-00135] [Medline: 19622511]

18. McGrath PJ, Walco GA, Turk DC, Dworkin RH, Brown MT, Davidson K, PedIMMPACT. Core outcome domains and measures for pediatric acute and chronic/recurrent pain clinical trials: PedIMMPACT recommendations. J Pain 2008 Sep;9(9):771-783. [doi: 10.1016/j.jpain.2008.04.007] [Medline: 18562251]

19. Dworkin RH, Turk DC, Farrar JT, Haythornthwaite JA, Jensen MP, Katz NP, IMMPACT. Core outcome measures for chronic pain clinical trials: IMMPACT recommendations. Pain 2005 Jan;113(1-2):9-19. [doi: 10.1016/j.pain.2004.09.012] [Medline: 15621359$]$

20. Landis JR, Koch GG. The measurement of observer agreement for categorical data. Biometrics 1977 Mar;33(1):159-174. [doi: 10.2307/2529310] [Medline: $\underline{843571]}$

21. Wood C, von Baeyer CL, Falinower S, Moyse D, Annequin D, Legout V. Electronic and paper versions of a faces pain intensity scale: concordance and preference in hospitalized children. BMC Pediatr 2011 Oct 12;11:87 [FREE Full text] [doi: 10.1186/1471-2431-11-87] [Medline: 21989306]

22. Castarlenas E, Sánchez-Rodríguez E, Vega RD, Roset R, Miró J. Agreement between verbal and electronic versions of the numerical rating scale (NRS-11) when used to assess pain intensity in adolescents. Clin J Pain 2015 Mar;31(3):229-234. [doi: 10.1097/AJP.0000000000000104] [Medline: 24699160]

23. Higgins JP, Thompson SG, Deeks JJ, Altman DG. Measuring inconsistency in meta-analyses. Br Med J 2003 Sep 6;327(7414):557-560 [FREE Full text] [doi: 10.1136/bmj.327.7414.557] [Medline: 12958120]

24. Stinson JN, Stevens BJ, Feldman BM, Streiner D, McGrath PJ, Dupuis A, et al. Construct validity of a multidimensional electronic pain diary for adolescents with arthritis. Pain 2008 Jun;136(3):281-292. [doi: 10.1016/j.pain.2007.07.002] [Medline: 17723279]

25. Allena M, Cuzzoni MG, Tassorelli C, Nappi G, Antonaci F. An electronic diary on a palm device for headache monitoring: a preliminary experience. J Headache Pain 2012 Oct;13(7):537-541 [FREE Full text] [doi: 10.1007/s10194-012-0473-2] [Medline: 22842873]

26. Athale N, Sturley A, Skoczen S, Kavanaugh A, Lenert L. A web-compatible instrument for measuring self-reported disease activity in arthritis. J Rheumatol 2004 Mar;31(2):223-228. [Medline: 14760788] 
27. Bandarian-Balooch S, Martin PR, McNally B, Brunelli A, Mackenzie S. Electronic-diary for recording headaches, triggers, and medication use: development and evaluation. Headache 2017 Nov;57(10):1551-1569. [doi: 10.1111/head.13184] [Medline: 28925498]

28. Bedson J, Hill J, White D, Chen Y, Wathall S, Dent S, et al. Development and validation of a pain monitoring app for patients with musculoskeletal conditions (the Keele pain recorder feasibility study). BMC Med Inform Decis Mak 2019 Jan 25;19(1):24 [FREE Full text] [doi: 10.1186/s12911-019-0741-z] [Medline: $\underline{\text { 30683106] }}$

29. Bishop FL, Lewis G, Harris S, McKay N, Prentice P, Thiel H, et al. A within-subjects trial to test the equivalence of online and paper outcome measures: the Roland Morris disability questionnaire. BMC Musculoskelet Disord 2010 Jun 8;11:113 [FREE Full text] [doi: 10.1186/1471-2474-11-113] [Medline: 20529332]

30. Blum D, Koeberle D, Omlin A, Walker J, von Moos R, Mingrone W, et al. Feasibility and acceptance of electronic monitoring of symptoms and syndromes using a handheld computer in patients with advanced cancer in daily oncology practice. Support Care Cancer 2014 Sep;22(9):2425-2434. [doi: 10.1007/s00520-014-2201-8] [Medline: 24705855]

31. Byrom B, Doll H, Muehlhausen W, Flood E, Cassedy C, McDowell B, et al. Measurement equivalence of patient-reported outcome measure response scale types collected using bring your own device compared to paper and a provisioned device: results of a randomized equivalence trial. Value Health 2018 May;21(5):581-589 [FREE Full text] [doi:

10.1016/j.jval.2017.10.008] [Medline: 29753356]

32. Chiu LY, Sun T, Ree R, Dunsmuir D, Dotto A, Ansermino JM, et al. The evaluation of smartphone versions of the visual analogue scale and numeric rating scale as postoperative pain assessment tools: a prospective randomized trial. Can J Anaesth 2019 Jun;66(6):706-715. [doi: 10.1007/s12630-019-01324-9] [Medline: 30796700]

33. Christie A, Dagfinrud H, Dale Y, Schulz T, Hagen KB. Collection of patient-reported outcomes; text messages on mobile phones provide valid scores and high response rates. BMC Med Res Methodol 2014 Apr 16;14:52 [FREE Full text] [doi: 10.1186/1471-2288-14-52] [Medline: 24735061]

34. Cook AJ, Roberts DA, Henderson MD, van Winkle LC, Chastain DC, Hamill-Ruth RJ. Electronic pain questionnaires: a randomized, crossover comparison with paper questionnaires for chronic pain assessment. Pain 2004 Jul;110(1-2):310-317. [doi: 10.1016/j.pain.2004.04.012] [Medline: 15275781]

35. Cunha-Miranda L, Santos H, Miguel C, Silva C, Barcelos F, Borges J, et al. Validation of Portuguese-translated computer touch-screen questionnaires in patients with rheumatoid arthritis and spondyloarthritis, compared with paper formats. Rheumatol Int 2015 Dec;35(12):2029-2035. [doi: 10.1007/s00296-015-3347-5] [Medline: 26346588]

36. Fanciullo GJ, Cravero JP, Mudge BO, McHugo GJ, Baird JC. Development of a new computer method to assess children's pain. Pain Med 2007 Oct;8(Suppl 3):S121-S128. [doi: 10.1111/j.1526-4637.2007.00376.x] [Medline: 17877522]

37. Freynhagen R, Baron R, Tölle T, Stemmler E, Gockel U, Stevens M, et al. Screening of neuropathic pain components in patients with chronic back pain associated with nerve root compression: a prospective observational pilot study (MIPORT). Curr Med Res Opin 2006 Mar;22(3):529-537. [doi: 10.1185/030079906X89874] [Medline: 16574036]

38. Gaertner J, Elsner F, Pollmann-Dahmen K, Radbruch L, Sabatowski R. Electronic pain diary: a randomized crossover study. J Pain Symptom Manage 2004 Sep;28(3):259-267 [FREE Full text] [doi: 10.1016/j.jpainsymman.2003.12.017] [Medline: 15336338 ]

39. Garcia-Palacios A, Herrero R, Belmonte MA, Castilla D, Guixeres J, Molinari G, et al. Ecological momentary assessment for chronic pain in fibromyalgia using a smartphone: a randomized crossover study. Eur J Pain 2014 Jul;18(6):862-872. [doi: 10.1002/j.1532-2149.2013.00425.x] [Medline: 24921074]

40. Heiberg T, Kvien TK, Dale O, Mowinckel P, Aanerud GJ, Songe-Møller AB, et al. Daily health status registration (patient diary) in patients with rheumatoid arthritis: a comparison between personal digital assistant and paper-pencil format. Arthritis Rheum 2007 Apr 15;57(3):454-460 [FREE Full text] [doi: 10.1002/art.22613] [Medline: 17394232]

41. Hofstedt O, di Giuseppe D, Alenius G, Stattin N, Forsblad-D'Elia H, Ljung L. Comparison of agreement between internet-based registration of patient-reported outcomes and clinic-based paper forms within the Swedish rheumatology quality register. Scand J Rheumatol 2019 Jul;48(4):326-330. [doi: 10.1080/03009742.2018.1551964] [Medline: 30758242]

42. Jaatun EA, Hjermstad MJ, Gundersen OE, Oldervoll L, Kaasa S, Haugen DF, European Palliative Care Research Collaborative (EPCRC). Development and testing of a computerized pain body map in patients with advanced cancer. J Pain Symptom Manage 2014 Jan;47(1):45-56 [FREE Full text] [doi: 10.1016/j.jpainsymman.2013.02.025] [Medline: 23856098]

43. Jamison RN, Gracely RH, Raymond SA, Levine JG, Marino B, Herrmann TJ, et al. Comparative study of electronic vs paper VAS ratings: a randomized, crossover trial using healthy volunteers. Pain 2002 Sep;99(1-2):341-347. [doi: 10.1016/s0304-3959(02)00178-1] [Medline: 12237213]

44. Jamison RN, Raymond SA, Slawsby EA, McHugo GJ, Baird JC. Pain assessment in patients with low back pain: comparison of weekly recall and momentary electronic data. J Pain 2006 Mar;7(3):192-199. [doi: 10.1016/j.jpain.2005.10.006] [Medline: $\underline{16516825]}$

45. Jonassaint CR, Shah N, Jonassaint J, de Castro L. Usability and feasibility of an mhealth intervention for monitoring and managing pain symptoms in sickle cell disease: the sickle cell disease mobile application to record symptoms via technology (SMART). Hemoglobin 2015;39(3):162-168. [doi: 10.3109/03630269.2015.1025141] [Medline: 25831427] 
46. Junker U, Freynhagen R, Längler K, Gockel U, Schmidt U, Tölle TR, et al. Paper versus electronic rating scales for pain assessment: a prospective, randomised, cross-over validation study with 200 chronic pain patients. Curr Med Res Opin 2008 Jun;24(6):1797-1806. [doi: 10.1185/03007990802121059] [Medline: 18485269]

47. Khan J, Jibb L, Busse J, Gilron I, Choi S, Paul J, et al. Electronic versus traditional data collection: a multicenter randomized controlled perioperative pain trial. Canadian J Pain 2019 Jul 30;3(2):16-25. [doi: 10.1080/24740527.2019.1587584]

48. Kim CH, Chung CK, Choi Y, Shin H, Woo JW, Kim S, et al. The usefulness of a mobile device-based system for patient-reported outcomes in a spine outpatient clinic. Spine J 2016 Jul;16(7):843-850. [doi: 10.1016/j.spinee.2016.02.048] [Medline: 26961200]

49. Koho P, Aho S, Kautiainen H, Pohjolainen T, Hurri H. Test-retest reliability and comparability of paper and computer questionnaires for the Finnish version of the Tampa Scale of Kinesiophobia. Physiotherapy 2014 Dec;100(4):356-362. [doi: 10.1016/j.physio.2013.11.007] [Medline: 24679373]

50. Kvien TK, Mowinckel P, Heiberg T, Dammann KL, Dale O, Aanerud GJ, et al. Performance of health status measures with a pen based personal digital assistant. Ann Rheum Dis 2005 Oct;64(10):1480-1484 [FREE Full text] [doi: 10.1136/ard.2004.030437] [Medline: $\underline{15843456]}$

51. MacKenzie H, Thavaneswaran A, Chandran V, Gladman DD. Patient-reported outcome in psoriatic arthritis: a comparison of web-based versus paper-completed questionnaires. J Rheumatol 2011 Dec;38(12):2619-2624. [doi: 10.3899/jrheum.110165] [Medline: 22045844]

52. Marceau LD, Link C, Jamison RN, Carolan S. Electronic diaries as a tool to improve pain management: is there any evidence? Pain Med 2007 Oct;8(Suppl 3):S101-S109. [doi: 10.1111/j.1526-4637.2007.00374.x] [Medline: 17877520]

53. Marceau LD, Link CL, Smith LD, Carolan SJ, Jamison RN. In-clinic use of electronic pain diaries: barriers of implementation among pain physicians. J Pain Symptom Manage 2010 Sep;40(3):391-404 [FREE Full text] [doi:

10.1016/j.jpainsymman.2009.12.021] [Medline: 20580526]

54. Matthews M, Rathleff MS, Vicenzino B, Boudreau SA. Capturing patient-reported area of knee pain: a concurrent validity study using digital technology in patients with patellofemoral pain. PeerJ 2018;6:e4406 [FREE Full text] [doi: 10.7717/peerj.4406] [Medline: 29568700]

55. Neudecker J, Raue W, Schwenk W. High correlation but inadequate point-to-point agreement, between conventional mechanical and electronical visual analogue scale for assessment of acute postoperative pain after general surgery. Acute Pain 2006 Dec;8(4):175-180. [doi: 10.1016/j.acpain.2006.08.043]

56. Palermo TM, Valenzuela D, Stork PP. A randomized trial of electronic versus paper pain diaries in children: impact on compliance, accuracy, and acceptability. Pain 2004 Mar;107(3):213-219. [doi: 10.1016/j.pain.2003.10.005] [Medline: 14736583]

57. Pawar SG, Ramani PS, Prasad A, Dhar A, Babhulkar SS, Bahurupi YA. Software version of Roland Morris disability questionnaire for outcome assessment in low back pain. Neurol Res 2017 Apr;39(4):292-297. [doi: 10.1080/01616412.2017.1297555] [Medline: 28337948]

58. Ritter P, Lorig K, Laurent D, Matthews K. Internet versus mailed questionnaires: a randomized comparison. J Med Internet Res 2004 Sep 15;6(3):e29 [FREE Full text] [doi: 10.2196/jmir.6.3.e29] [Medline: 15471755]

59. Rolfson O, Salomonsson R, Dahlberg LE, Garellick G. Internet-based follow-up questionnaire for measuring patient-reported outcome after total hip replacement surgery-reliability and response rate. Value Health 2011;14(2):316-321 [FREE Full text] [doi: 10.1016/j.jval.2010.08.004] [Medline: 21402299]

60. Saleh KJ, Radosevich DM, Kassim RA, Moussa M, Dykes D, Bottolfson H, et al. Comparison of commonly used orthopaedic outcome measures using palm-top computers and paper surveys. J Orthop Res 2002 Nov;20(6):1146-1151 [FREE Full text] [doi: 10.1016/S0736-0266(02)00059-1] [Medline: 12472221]

61. Sánchez-Rodríguez E, de la Vega R, Castarlenas E, Roset R, Miró J. An app for the assessment of pain intensity: validity properties and agreement of pain reports when used with young people. Pain Med 2015 Oct;16(10):1982-1992. [doi: 10.1111/pme.12859] [Medline: 26176297]

62. Serif T, Ghinea G. Recording of time-varying back-pain data: a wireless solution. IEEE Trans Inf Technol Biomed 2005 Sep;9(3):447-458. [doi: 10.1109/titb.2005.847514] [Medline: 16167699]

63. Stinson JN, Connelly M, Jibb LA, Schanberg LE, Walco G, Spiegel LR, et al. Developing a standardized approach to the assessment of pain in children and youth presenting to pediatric rheumatology providers: a Delphi survey and consensus conference process followed by feasibility testing. Pediatr Rheumatol Online J 2012 Apr 10;10(1):7 [FREE Full text] [doi: 10.1186/1546-0096-10-7] [Medline: 22490427]

64. Stomberg MW, Platon B, Widén A, Wallner I, Karlsson O. Health information: what can mobile phone assessments add? Perspect Health Inf Manag 2012;9:1-10 [FREE Full text] [Medline: 23209453]

65. Stone AA, Broderick JE, Schwartz JE, Shiffman S, Litcher-Kelly L, Calvanese P. Intensive momentary reporting of pain with an electronic diary: reactivity, compliance, and patient satisfaction. Pain 2003 Jul;104(1-2):343-351. [doi: 10.1016/s0304-3959(03)00040-x] [Medline: 12855344]

66. Sun T, West N, Ansermino JM, Montgomery CJ, Myers D, Dunsmuir D, et al. A smartphone version of the faces pain scale-revised and the color analog scale for postoperative pain assessment in children. Paediatr Anaesth 2015 Dec;25(12):1264-1273. [doi: 10.1111/pan.12790] [Medline: 26507916] 
67. Suso-Ribera C, Castilla D, Zaragozá I, Ribera-Canudas MV, Botella C, García-Palacios A. Validity, reliability, feasibility, and usefulness of pain monitor: a multidimensional smartphone app for daily monitoring of adults with heterogenous chronic pain. Clin J Pain 2018 Oct;34(10):900-908. [doi: 10.1097/AJP.0000000000000618] [Medline: 29659375]

68. Symonds T, Hughes B, Liao S, Ang Q, Bellamy N. Validation of the Chinese western Ontario and McMaster universities osteoarthritis index in patients from mainland china with osteoarthritis of the knee. Arthritis Care Res (Hoboken) 2015 Nov;67(11):1553-1560 [FREE Full text] [doi: 10.1002/acr.22631] [Medline: 26018634]

69. Theiler R, Alon E, Brugger S, Ljutow A, Mietzsch T, Müller D, et al. Evaluation of a standardized internet-based and telephone-based patient monitoring system for pain therapy with transdermal fentanyl. Clin J Pain 2007;23(9):804-811. [doi: 10.1097/AJP.0b013e3181565d04] [Medline: 18075409]

70. van den Kerkhof EG, Goldstein DH, Lane J, Rimmer MJ, van Dijk JP. Using a personal digital assistant enhances gathering of patient data on an acute pain management service: a pilot study. Can J Anaesth 2003 Apr;50(4):368-375. [doi:

10.1007/BF03021034] [Medline: 12670814]

71. van den Kerkhof EG, Goldstein DH, Rimmer MJ, Tod DA, Lee HK. Evaluation of hand-held computers compared to pen and paper for documentation on an acute pain service. Acute Pain 2004 Dec;6(3-4):115-121. [doi:

10.1016/j.acpain.2004.07.001]

72. Wæhrens EE, Amris K, Bartels EM, Christensen R, Danneskiold-Samsøe B, Bliddal H, et al. Agreement between touch-screen and paper-based patient-reported outcomes for patients with fibromyalgia: a randomized cross-over reproducibility study. Scand J Rheumatol 2015;44(6):503-510. [doi: 10.3109/03009742.2015.1029517] [Medline: 26114582]

73. Sanchez MA, Rabin BA, Gaglio B, Henton M, Elzarrad MK, Purcell P, et al. A systematic review of eHealth cancer prevention and control interventions: new technology, same methods and designs? Transl Behav Med 2013 Dec;3(4):392-401 [FREE Full text] [doi: 10.1007/s13142-013-0224-1] [Medline: 24294327]

74. Steele L, Lade H, McKenzie S, Russell TG. Assessment and diagnosis of musculoskeletal shoulder disorders over the internet. Int J Telemed Appl 2012;2012:945745 [FREE Full text] [doi: 10.1155/2012/945745] [Medline: 23193395]

75. Connelly M, Neville K. Comparative prospective evaluation of the responsiveness of single-item pediatric pain-intensity self-report scales and their uniqueness from negative affect in a hospital setting. J Pain 2010 Dec;11(12):1451-1460. [doi: 10.1016/j.jpain.2010.04.011] [Medline: 20646966]

76. Gagliese L, Katz J. Age differences in postoperative pain are scale dependent: a comparison of measures of pain intensity and quality in younger and older surgical patients. Pain 2003 May;103(1-2):11-20. [doi: 10.1016/s0304-3959(02)00327-5] [Medline: 12749954]

77. Fleischmann R, Decker A, Kraft A, Mai K, Schmidt S. Mobile electronic versus paper case report forms in clinical trials: a randomized controlled trial. BMC Med Res Methodol 2017 Dec 1;17(1):153 [FREE Full text] [doi: 10.1186/s12874-017-0429-y] [Medline: 29191176]

78. Walther B, Hossin S, Townend J, Abernethy N, Parker D, Jeffries D. Comparison of electronic data capture (EDC) with the standard data capture method for clinical trial data. PLoS One 2011;6(9):e25348 [FREE Full text] [doi:

10.1371/journal.pone.0025348] [Medline: 21966505]

79. Walker I, Sigouin C, Sek J, Almonte T, Carruthers J, Chan A, et al. Comparing hand-held computers and paper diaries for haemophilia home therapy: a randomized trial. Haemophilia 2004 Nov;10(6):698-704. [doi: 10.1111/j.1365-2516.2004.01046.x] [Medline: 15569164$]$

\section{Abbreviations \\ ICC: intraclass correlation coefficient \\ NRS: Numerical Rating Scale \\ PDA: personal digital assistant \\ VAS: Visual Analog Scale}

Edited by G Eysenbach; submitted 16.10.19; peer-reviewed by S Haghayegh, E Sadeghi-Demneh; comments to author 14.11.19;
revised version received 21.01.20; accepted 22.03.20; published 16.06.20
Please cite as:
Jibb LA, Khan JS, Seth P, Lalloo C, Mulrooney L, Nicholson K, Nowak DA, Kaur H, Chee-A-Tow A, Foster J, Stinson JN
Electronic Data Capture Versus Conventional Data Collection Methods in Clinical Pain Studies: Systematic Review and Meta-Analysis
J Med Internet Res 2020;22(6):e16480
URL: $\underline{\text { https://www.jmir.org/2020/6/e16480 }}$
doi: $\underline{10.2196 / 16480}$
PMID: $\underline{32348259}$


CLindsay A Jibb, James S Khan, Puneet Seth, Chitra Lalloo, Lauren Mulrooney, Kathryn Nicholson, Dominik A Nowak, Harneel Kaur, Alyssandra Chee-A-Tow, Joel Foster, Jennifer N Stinson. Originally published in the Journal of Medical Internet Research (http://www.jmir.org), 16.06.2020. This is an open-access article distributed under the terms of the Creative Commons Attribution License (https://creativecommons.org/licenses/by/4.0/), which permits unrestricted use, distribution, and reproduction in any medium, provided the original work, first published in the Journal of Medical Internet Research, is properly cited. The complete bibliographic information, a link to the original publication on http://www.jmir.org/, as well as this copyright and license information must be included. 